Pacific Journal of Mathematic 


\title{
$N$-DIMENSIONAL AREA AND CONTENT IN MINKOWSKI SPACES
}

\author{
R. D. Holmes AND A. C. Thompson
}

A definition of content in Minkowski spaces (of any finite dimension) is given which implies that the surface of the unit ball and that of the dual ball are equal. Various consequences of this definition, including the solution to the isoperimetric problem, are explored. Numerous examples and some unsolved problems are given in the last two sections.

1. Introduction. The original motivation for this investigation came from the work of J. J. Schäffer on geometrical constants associated with the unit ball in a normed linear space. He and $\mathrm{K}$. Sundaresan [15] showed that, if a normed linear space is nonreflexive, then the "girth" of the unit ball is 4 . In the paper [13], in which girth and inner diameter were first defined, he also showed that, if $\mathscr{Z}$ is 2-dimensional, the girth lies in the interval $[6,8]$. It was these latter inequalities which suggested the consideration of higher dimensional parameters (area, volume, etc.) and, specifically, to ask for bounds for the surface area of the unit ball in a 3-dimensional space. This problem is still, as far as we know, unsolved (see Problem 7.9 below); but it requires, first of all, a definition of area. Such a definition is the first aim of the present paper, and our proposal is contained in $\S 2$. The second aim is to investigate the solution to the isoperimetric problem which results from our definition of area; this is contained in $\S 4$. Throughout this paper, but especially in $\S 4$, we rely on the work of $H$. Busemann and his school [3], [4], [5], [6]. A more detailed summary of the contents of each section will be given after we have explained our notation.

Throughout, we will be concerned with finite-dimensional real linear spaces upon which we impose a variety of norms. Script roman letters will be used for affine sets $-\mathscr{X}, \mathscr{Y}, \mathscr{Z}$ for spaces and subspaces, $\mathscr{F}, \mathscr{C}, \mathscr{H}$ for hyperplanes. Small roman letters will be used for vectors $-p, q, \cdots, z$ in the space $\mathscr{P}$ and $a, b, \cdots$, $h$ in the dual space (i.e., linear functionals on $\mathscr{P}$ ). For obvious reasons it is not always possible to be completely systematic about this. Letters from the middle of the alphabet $-i, j, \cdots, n-$ will be reserved for natural numbers. Capital roman letters $-A, B, \cdots-$ will be used to denote convex sets, while nonconvex sets (and surfaces in particular), will be denoted by capital greek letters 
( $d \Sigma$ is used for a surface area element). Small greek letters will be used for real numbers and nonlinear real-valued functions. Distinguished here, are the symbols $\mu$ and $\varepsilon$ which are used to denote Minkowskian and Euclidean content respectively (including the norms); the dimension of the content being inferred from the context. The $n$-dimensional Euclidean unit ball is denoted by $E_{n}$ and $\varepsilon_{n}=\varepsilon\left(E_{n}\right)=\pi^{n / 2} \Gamma(n+2 / 2)^{-1}$. Finally, linear transformations are shown by bold face capitals.

Since all norms on $\mathscr{P}$ are topologically equivalent, the set of continuous linear functionals on $\mathscr{X}$ (the dual space) is independent of the choice of norm (and is, of course, a linear space of the same dimension as $\mathscr{X}$ ). This space will be denoted by $\mathscr{X}^{*}$. Given a convex set $B$ in $\mathscr{C}$, the polar $B^{0}$ of $B$ is defined by

$$
B^{0}=\left\{f \in \mathscr{Z}^{*}|| f(x) \mid \leqq 1, \quad \forall x \in B\right\} .
$$

As the space $\mathscr{P}$ is not explicitly mentioned in the polar notation, it frequently has to be inferred from the context. In particular, when $B$ is the unit ball with respect to a norm $\mu$, then $B^{0}$ is the unit ball for the dual norm $\mu^{0}$.

In this paper, we are not concerned with questions of measurability. In a finite-dimensional linear space, it is always possible to introduce an auxiliary Euclidean metric and consequently a Lebesgue measure, which is determined up to a scalar multiple. The question is which multiple best fits the Minkowskian geometry. Choosing a multiple is equivalent to assigning a number $\gamma(B)=$ $\mu_{B}(B)$ to be the Minkowski content of the unit ball $B$. Thus $\gamma$ is to be a real-valued function defined on all centrally symmetric convex bodies (of any finite dimension). Section 2 is concerned with the problem of choosing a function $\gamma$ and it is shown that it is possible to define $\gamma$ in a way which fits the geometry in the sense that

$$
\mu_{B}(\partial B)=\mu_{B^{0}}^{0}\left(\partial B^{0}\right) .
$$

This is a special case of Theorem 2.11 which is, perhaps, the main result of the paper.

Though $\gamma$ and $\mu$ behave well, certain constants depending on the dimension keep occurring. By letting $\rho$ be an appropriate multiple of $\mu$ (depending on the dimension) we show in $\S 3$ that $\rho$ has a number of pleasing algebraic properties.

Section 4 deals with the isoperimetric problem. Here, the main result is Theorem 4.5 which asserts that, if the unit ball is a convex polytope, then so also is the solution to the isoperimetric problem. We are also able to give formulas for constructing the solution from the given ball. 
In $\S 5$ we briefly discuss the ideas of Busemann on normality and transversality as applied in relation to our area function $\gamma$. The next section is concerned with examples in $\mathscr{R}^{3}$. In particular, we give evidence for the view that $\gamma$ is uniquely determined by the condition (1.1). There are also a number of specific solutions to the isoperimetric problem. As far as we know, this has not been done for other area functions. We have also computed the solution in $n$-dimensions when the unit ball is a cube or its dual, but the computations are too tedious to include here.

As stated above, our original problem - to give exact bounds for $\mu_{B}(\partial B)$ in $\mathscr{R}^{3}-$ is still unsolved. This, and a number of other, as yet, unsolved problems arising from these investigations, are collected in $\S 7$.

2. General theory, the function $\gamma$. Given an $n$-dimensional Minkowski space $\mathscr{C}$ with norm $\mu$ derived from the unit ball $B$, it is always possible to introduce an auxiliary Euclidean norm $\varepsilon$ and, consequently, Lebesgue measure. This measure, is, however, only determined up to a scalar multiple, and the question arises of normalizing it in a suitable way. One may either introduce $\varepsilon$ in a more or less canonical way - for example, by taking as unit ball the largest (in $n$-dimensional content) ellipsoid inscribed in $B$ or the smallest ellipsoid circumcribed to $B$ - or assign a number $\mu(B)$ as the $n$-dimensional content of the unit ball. We shall adopt the latter approach; thus, if $\Delta$ is some measurable domain in $\mathscr{X}$ and $\varepsilon$ is a fixed Euclidean norm:

$$
\mu(\Delta)=\frac{\varepsilon(\Delta)}{\varepsilon(B)} \mu(B)=\sigma(\mathscr{X}) \varepsilon(\Delta)
$$

where $\sigma(\mathscr{Z})$ denotes the ratio $\mu(B) / \varepsilon(B)$.

The situation becomes more interesting when one considers the measure in subspaces of $\mathscr{C}^{\circ}$, for, as the space has in general few isometries, it is possible for this ratio to vary from subspace to subspace. For a fixed Euclidean norm $\varepsilon$ on $\mathscr{X}, \sigma$ is then a function from all subspaces of $\mathscr{P}$ to the positive reals, $\mathscr{R}^{+}$(the ratio being taken with respect to the unit ball in the subspace and the inherited Euclidean norm). Of particular importance are the subspaces of codimension 1 determined by linear functionals in $\mathscr{Z}^{*}$. If $f \in \mathscr{X}^{*}$, let $f^{\perp}=\{x \in \mathscr{X} \mid f(x)=0\}$, then we have

$$
\sigma\left(f^{\perp}\right)=\frac{\mu\left(B \cap f^{\perp}\right)}{\varepsilon\left(B \cap f^{\perp}\right)} .
$$

Then, if $\Delta$ is a measurable domain in a hyperplane $\mathscr{H}=\{x \epsilon$ 
$\mathscr{Z} \mid f(x)=\alpha\}$, we can, as measure is translation invariant, translate $\Delta$ to $\Delta^{\prime} \subseteq f^{\perp}$ so that

$$
\mu(\Delta)=\mu\left(\Delta^{\prime}\right)=\frac{\varepsilon\left(\Delta^{\prime}\right)}{\varepsilon\left(B \cap f^{\perp}\right)} \mu\left(B \cap f^{\perp}\right)=\sigma\left(f^{\perp}\right) \varepsilon(\Delta) .
$$

In the sequel, we shall be using a variety of unit balls $B$ and their restrictions to subspaces. To indicate that $\mu$ depends on $B$ we shall write $\mu_{B}(\Delta)$. However, in order to simplify the notation, we make the following definition:

Definition 2.1. Let $\mathscr{X}$ be a Minkowski space with unit ball $B$ and $\mathscr{Y}$ be an $m$-dimensional subspace of $\mathscr{C}$ with unit ball $C=$ $B \cap \mathscr{Y}$, then define

$$
\gamma_{\mathscr{R}}(C)=\mu_{B}(C)
$$

(the $m$-dimensional measure of the unit ball $C$ in the space $\mathscr{X}$ ). If $\mathscr{Y}=\mathscr{X}, C=B$ then we denote $\gamma_{\mathscr{X}}(B)$ simply by $\gamma(B)$. Our first concern will be the nature of these functions $\gamma_{\mathscr{C}}$. We note that very little of what follows depends on the symmetry of the metric (i.e., the unit ball). We begin by listing the commonly accepted axioms for the functions $\gamma_{x}$.

Axioms 2.2.

(a) $\gamma_{\mathscr{C}}$ is independent of the space $\mathscr{X}$, i.e., $\gamma_{\mathscr{X}}(C)$ depends only on the shape of the ball $C$ and not on that of the ball $B$ in the larger space in which it is embedded.

(b) $\gamma$ is continuous as a function from the collection of compact subsets of $\mathscr{R}^{n}$ with the Hausdorff metric to $\mathscr{R}^{+}$.

(c) $\gamma$ is invariant under nonsingular linear transformations $\boldsymbol{L}$ from $\mathscr{R}^{n}$ to $\mathscr{R}^{n}$.

(d) If $E_{n}$ is an $n$-dimensional Euclidean unit ball (i.e., an ellipsoid) then,

$$
\gamma\left(E_{n}\right)=\varepsilon\left(E_{n}\right)=\varepsilon_{n}=\pi^{n / 2} \Gamma\left(\frac{n+2}{2}\right)^{-1} .
$$

There is considerable discussion of such functions $\gamma$ in the literature, most notably by $\mathrm{H}$. Minkowski and later by $\mathrm{H}$. Busemann and his school. An elementary account is given in the last chapter of the book [2] by R.V. Benson.

In view of Axiom 2.2 (a) we shall, from now on, write $\gamma(C)$ for $\gamma_{Z}(C)$ since the space in which $C$ is embedded does not affect the value of $\gamma(C)$. This makes it clear that $\gamma$ is a function from the set of all (centrally symmetric) closed, bounded, convex bodies 
in $\mathscr{R}^{n}$ ( $n$ arbitrary) to $\mathscr{R}^{+}$.

The simplest way to achieve (a) $\sim(d)$ is to make $\gamma$ constant on $\mathscr{R}^{n}$

$$
\gamma(B)=\varepsilon_{n}=\frac{\pi^{n / 2}}{\Gamma\left(\frac{n+2}{2}\right)}
$$

These numbers appear somewhat arbitrary and it was hoped that a function more intimately connected with the geometry (i.e., the metric) could be constructed. One possibility in $\mathscr{R}^{2}$ is $\gamma(B)=$ $(1 / 2) \mu(\partial B)$ and, inductively,

$$
\gamma(B)=\frac{1}{n} \mu(\partial B)
$$

for $B \subseteq \mathscr{R}^{n}$. Another possibility considered in the literature, is to choose $\gamma$ is such a way that the minimum $n$-dimensional measure of a parallelepiped circumcribed about $B$ is equal to $2^{n}$.

Recently, J. J. Schäffer [14] (see also [16]) has shown that, if $\mathscr{X}$ is a 2-dimensional space with unit ball $B$, the

$$
\mu_{B}(\partial B)=\mu_{B^{0}}\left(\partial B^{0}\right) \text {. }
$$

The main purpose of this investigation is to show that it is possible to define $\gamma$ in such a way that (2.7) holds in general (see Corollary 2.12 below) and to examine the consequences of this definition. Our first observation is that none of the above candidates for $\gamma$ satisfies (2.7) as a simple calculation with the cube and octahedron in $\mathscr{R}^{3}$ will demonstrate. The second is that extensive calculations in $\mathscr{R}^{3}$ (see §6) surprized us, not only by not yielding a contradiction, but by suggesting that Axioms 2.2 and equation (2.7) together uniquely determine $\gamma$ (although we have been unable to prove this). Our calculations suggested the following definition for $\gamma$.

Definition 2.3. Let $C$ be a fixed $n$-dimensional Minkowski unit ball in $\mathscr{R}^{n}$ and assign it an (arbitrary) value $\gamma(C)$. For each $n$ dimensional ball $B$ in $\mathscr{R}^{n}$ we require that the $n$-dimensional content $\gamma(B)$ of $B$ satisfy:

$$
\gamma(B)=\frac{\mu_{C}(B) \mu_{0^{0}}\left(B^{0}\right)}{\gamma(C)}
$$

REMARK 2.4. Putting $B=C$ in (2.8) implies that we must have $\gamma\left(C^{0}\right)=\gamma(C)$. After applying the formulae for these measures it is easily shown that $\gamma$ as defined above satisfies Axioms 2.2 (a), (b) and (c). 
Proposition 2.5. If $\gamma$ also satisfies $\gamma\left(E_{n}\right)=\varepsilon_{n}$, then we have, for each ball $B$ in $\mathscr{R}^{n}$ and any Euclidean norm $\varepsilon$ on $\mathscr{R}^{n}$

$$
\gamma(B)=\varepsilon_{n}^{-1} \varepsilon(B) \varepsilon\left(B^{0}\right) \text {. }
$$

Proof. Noting that $\mu_{C}(B)=(\varepsilon(B) / \varepsilon(C)) \gamma(C)$ and $\mu_{C^{0}}\left(B^{0}\right)=\left(\varepsilon\left(B^{0}\right) /\right.$ $\left.\varepsilon\left(C^{0}\right)\right) \gamma\left(C^{0}\right)$ and substituting these values in (2.8) we obtain,

$$
\begin{aligned}
\gamma(B) & =\gamma(C)^{-1} \varepsilon(B) \varepsilon(C)^{-1} \gamma(C) \varepsilon\left(B^{0}\right) \varepsilon\left(C^{0}\right)^{-1} \gamma\left(C^{0}\right) \\
& =\varepsilon(B) \varepsilon\left(B^{0}\right) \varepsilon(C)^{-1} \varepsilon\left(C^{0}\right)^{-1} \gamma\left(C^{0}\right) .
\end{aligned}
$$

Letting $B=E_{n}$ in (2.10), we have

$$
\varepsilon_{n}=\gamma\left(E_{n}\right)=\varepsilon_{n}^{2} \gamma\left(C^{0}\right) \varepsilon(C)^{-1} \varepsilon\left(C^{0}\right) \text { which yields } \varepsilon_{n}=\gamma\left(C^{0}\right)^{-1} \varepsilon(C) \varepsilon\left(C^{0}\right) .
$$

Substituting in (2.10) gives the desired result.

REMARK 2.6. The function $\varepsilon(B) \varepsilon\left(B^{0}\right)$ has been of interest in the geometry of numbers. Cassels [7, p. 118] points out that, in $\mathscr{R}^{2}$, upper and lower bounds have been established by K. Mahler [9] and L. Santaló [12], these bounds being attained for parallelograms $Q$ and ellipses $D$.

$$
\gamma(Q)=\frac{8}{\pi} \leqq \gamma(B) \leqq \pi=\gamma(D)
$$

Santaló also showed that the upper bound in $\mathscr{R}^{n}$ is attained when $B$ is a Euclidean ball, but the precise lower bound in $\mathscr{R}^{n}$ is unknown though Mahler has conjectured that it is attained by the $n$-dimensional cube. It is known that in $\mathscr{R}^{n},(B a m b a h$ [1], and [7, p. 118])

$$
\varepsilon(B) \varepsilon\left(B^{0}\right) \geqq(n !)^{-2} 4^{n} .
$$

Henceforth, all Minkowski contents will be derived from (2.9) (by the proposition, equivalent to Definition 2.3 plus Axiom 2.2(d)). The definition was formulated in a way to avoid any dependence on a Euclidean metric, but we use (2.9) for calculation because of the great number of well known formulas for computing $\varepsilon(B)$ and $\varepsilon\left(B^{0}\right)$.

An easily established property of $\gamma$ is

Proposition 2.7. Let $A$ and $B$ be two balls in $\mathscr{R}^{n}$, then

$$
\mu_{A}(B)=\mu_{B} \circ\left(A^{0}\right) \text {. }
$$

Proof. Let $\varepsilon$ be a Euclidean metric on $\mathscr{R}^{n}$, then 


$$
\begin{aligned}
\mu_{A}(B)=\frac{\varepsilon(B)}{\varepsilon(A)} \gamma(A) & =\frac{\varepsilon(B)}{\varepsilon(A)} \frac{\varepsilon(A) \varepsilon\left(A^{0}\right)}{\varepsilon_{n}}=\frac{\varepsilon(B) \varepsilon\left(A^{0}\right)}{\varepsilon_{n}}=\frac{\varepsilon\left(A^{0}\right)}{\varepsilon\left(B^{0}\right)} \frac{\varepsilon\left(B^{0}\right) \varepsilon(B)}{\varepsilon_{n}} \\
& =\frac{\varepsilon\left(A^{0}\right)}{\varepsilon\left(B^{0}\right)} \gamma\left(B^{0}\right)=\mu_{B^{0}}\left(A^{0}\right) .
\end{aligned}
$$

We now turn our attention to the function $\sigma$. Let $\mathscr{Y}$ be an $m$-dimensional subspace of the Minkowski space $\mathscr{X}$ with unit ball $B$. Then the unit ball in $\mathscr{Y}$ is given by $\mathscr{Y} \cap B$ and we have, by (2.9),

$$
\sigma(\mathscr{Y})=\frac{\mu(\mathscr{Y} \cap B)}{\varepsilon(\mathscr{Y} \cap B)}=\frac{\gamma(\mathscr{Y} \cap B)}{\varepsilon(\mathscr{Y} \cap B)}=\frac{\varepsilon\left((\mathscr{Y} \cap B)^{0}\right)}{\varepsilon_{m}} .
$$

This shows the need for a characterization of $(\mathscr{Y} \cap B)^{0}$ such as is given by the following goemetrical result.

Proposition 2.8 (MacMullen and Shephard [10], p. 70). Let $B$ be a convex body in $\mathscr{E}^{n}$ with 0 as an interior point, let $\mathscr{Y}$ be a subspace of $\mathscr{E}^{n}$ and let $\boldsymbol{P}$ be the orthogonal projection along $\mathscr{Y}^{\perp}=$ $\left\{f \in\left(\mathscr{C}^{n}\right)^{*} \mid f(\mathscr{Y})=0\right\}$. Then $(B \cap \mathscr{Y})^{0}=\boldsymbol{P}\left(B^{0}\right)$.

The proof given in [10] is for polytopes buta continuity argument will extend the result to convex bodies. We have used a notation which keeps the distinction between the space $\mathscr{C}^{n}$ and its dual clear. As a particular case, let $\mathscr{Y}=f^{\perp}$ for $f \in \mathscr{E}^{*}$. Then $\mathscr{Y}^{\perp}$ is the linear space spanned by $f$ and, if $\boldsymbol{P}_{f}$ denotes the orthogonal projection along $f$, we have $\left(B \cap f^{\perp}\right)^{0}=\boldsymbol{P}_{f}\left(B^{0}\right)$. Consequently:

$$
\sigma\left(f^{\perp}\right)=\varepsilon_{n-1}^{-1} \varepsilon\left(\boldsymbol{P}_{f}\left(B^{0}\right)\right) .
$$

If $\Sigma$ is an $(n-1)$-dimensional hypersurface in $\mathscr{E}^{n}$ we will denote the $(n-1)$-dimensional Euclidean "area element" of $\Sigma$ at $x$ by $d \Sigma_{x}$ and the Euclidean unit normal to $\Sigma$ at the point $x$ by $\widetilde{x}\left(\right.$ in $\left.\left(\mathscr{E}^{n}\right)^{*}\right)$.

LEMMA 2.9. If $f$ is a Euclidean unit vector in $\mathscr{Z}^{*}$, then

$$
\sigma\left(f^{\perp}\right)=\frac{1}{2 \varepsilon_{n-1}} \int_{\Sigma=\partial B^{0}}|f(\widetilde{g})| d \Sigma_{g} .
$$

Proof. As $B^{0}$ is convex, it has a unique tangent hyperplane almost everywhere. Thus $\widetilde{g}$ is defined almost everywhere and the integral in (2.13) exists. Let $\boldsymbol{P}=\boldsymbol{P}_{f}$ and $\Sigma^{\prime}=\boldsymbol{P}\left(B^{\circ}\right)$. Now consider a fixed point $g \in \Sigma$. The area element $d \Sigma_{P(g)}^{\prime}$ of $\Sigma^{\prime}$ at $\boldsymbol{P}(g)$ is the projection of $d \Sigma_{g}$ and is normal to $f$. Thus $d \Sigma_{P(g)}^{\prime}$ is $\pm d \Sigma_{g}$ times the cosine of the angle between $f$ and $\widetilde{g}$ (which is, of course, $f(\widetilde{g})$ ). 
In other words $d \Sigma_{P(g)}^{\prime}=|f(\widetilde{g})| d \Sigma_{g}$. As $B^{0}$ is convex $P(\Sigma)$ covers $\Sigma^{\prime}$ exactly twice, therefore

$$
\int_{\Sigma}|f(\widetilde{g})| d \Sigma_{g}=\int_{\Sigma} d \Sigma_{P(g)}^{\prime}=2 \int_{\Sigma^{\prime}} d \Sigma^{\prime}=2 \varepsilon\left(\Sigma^{\prime}\right) .
$$

By (2.12), $\sigma\left(f^{\perp}\right)=\left(1 / \varepsilon_{n-1}\right) \varepsilon\left(\boldsymbol{P}_{f}\left(B^{0}\right)\right)=\left(1 / \varepsilon_{n-1}\right) \varepsilon\left(\Sigma^{\prime}\right)$ which with (2.14) yields (2.13).

LEMMA 2.10. If $\Sigma$ is a surface in $\mathscr{X}$ then

$$
\mu(\Sigma)=\int_{\Sigma} \sigma\left(\tilde{x}^{\perp}\right) d \Sigma_{x}
$$

Proof. $\sigma(\mathscr{Y})$ is the ratio of Minkowski measure in $\mathscr{Y}$ (and in hyperplanes parallel to $\mathscr{Y}$ ) to Euclidean measure in $\mathscr{Y}$. Thus it is also the ratio of the Minkowski area element $d_{\mu} \Sigma$ to $d \Sigma$ when both are parallel to $\mathscr{Y}$. But, by definition, $\tilde{x}^{\perp}$ is the subspace of $\mathscr{P}$ parallel to the hyperplane tangent to $\Sigma$ at $x$ and hence to $d_{\mu} \Sigma$ and $d \Sigma$. Thus $\mu(\Sigma)=\int_{\Sigma} d_{\mu} \Sigma=\int_{\Sigma} \sigma\left(\widetilde{x}^{\perp}\right) d \Sigma$ as required.

We are now in a position to prove the main theorem.

THEOREM 2.11. Let $A$ and $B$ be two Minkowski unit balls in $\mathscr{Z}$, then

$$
\mu_{B}(\partial A)=\mu_{A} \circ\left(\partial B^{0}\right)
$$

Proof. By continuity, we may assume that all balls are strictly convex and sufficiently smooth. Denote $\partial A$ by $\Sigma$ and $\partial B^{\circ}$ by $\Sigma^{\prime}$ and choose a fixed Euclidean norm in $\mathscr{X}$. Let $x \in \Sigma$, then by (2.13), $\sigma\left(\widetilde{x}^{\perp}\right)=\left(1 / 2 \varepsilon_{n-1}\right) \int_{\Sigma^{\prime}}|\widetilde{x}(\widetilde{g})| d \Sigma_{g}^{\prime}$ which when substituted in (2.15) yields

$$
\mu_{B}(\partial A)=\frac{1}{2 \varepsilon_{n-1}} \int_{\Sigma} \int_{\Sigma_{1}}|\widetilde{x}(\widetilde{g})| d \Sigma_{g}^{\prime} d \Sigma_{x} .
$$

Similarly, $\sigma\left(\widetilde{g}^{\perp}\right)=\left(1 / 2 \varepsilon_{n-1}\right) \int_{\Sigma}|\widetilde{x}(\widetilde{g})| d \Sigma_{x}$ and

$$
\mu_{A^{0}}\left(\partial B^{0}\right)=\frac{1}{2 \varepsilon_{n-1}} \int_{\Sigma} \int_{\Sigma}|\widetilde{x}(\widetilde{g})| d \Sigma_{x} d \Sigma_{g}^{\prime}
$$

which, by comparison with (2.16), gives the desired equality.

CoRollary 2.12. If $B$ is a unit ball in $\mathscr{X}, \mu_{B}(\partial B)=\mu_{B^{0}}\left(\partial B^{0}\right)$. 
The idea for the proof of Theorem 2.11 comes from the proof of Cauchy's surface area formula as given in Eggleston ([8], p. 89). Indeed, since the symmetry of the unit ball is not used, we derive an analogue of Cauchy's formula as follows.

Corollary 2.13. If $C$ is a convex body in n-dimensional Minkowski space $\mathscr{X}^{\mathrm{P}}$ with unit ball $B$, then

$$
\mu_{B}(\partial C)=\frac{1}{\varepsilon_{n-1}} \int_{\Sigma=\partial B^{0}} \varepsilon\left(\boldsymbol{P}_{\tilde{g}}(C)\right) d \Sigma_{g} .
$$

Proof. In the same way that (2.14) was derived, we have $\varepsilon\left(\boldsymbol{P}_{\tilde{g}}(C)\right)=(1 / 2) \int_{\Sigma^{\prime}=\partial C}|\widetilde{x}(\widetilde{g})| d \Sigma_{x}^{\prime}$. Thus, from Lemmas 2.9 and 2.10,

$$
\begin{aligned}
& \frac{1}{\varepsilon_{n-1}} \int_{\partial B^{0}} \varepsilon\left(\boldsymbol{P}_{\tilde{g}}(C)\right) d \Sigma_{g}=\frac{1}{2 \varepsilon_{n-1}} \int_{\partial B^{0}} \int_{\partial C}|\widetilde{x}(\widetilde{g})| d \Sigma_{x}^{\prime} d \Sigma_{g} \\
& \quad=\int_{\partial C} \frac{1}{2 \varepsilon_{n-1}} \int_{\partial B^{0}}|\widetilde{x}(\widetilde{g})| d \Sigma_{g} d \Sigma_{x}^{\prime}=\int_{\partial C} \sigma\left(\widetilde{x}^{\perp}\right) d \Sigma_{x}^{\prime}=\mu(\partial C) .
\end{aligned}
$$

REMARK 2.14. If, in Theorem 2.11, $A$ and $B$ are assumed to be polytopes (rather then strictly convex and smooth), than the integrals become finite sums and $(2.16)$ can be replaced by

$$
\mu_{B}(\partial A)=\mu_{A^{0}}\left(\partial B^{0}\right)=\frac{1}{2 \varepsilon_{n-1}} \sum_{i=1}^{m} \sum_{j=1}^{k}\left|f_{i}\left(x_{j}\right)\right| \varepsilon\left(F_{i}\right) \varepsilon\left(X_{j}\right)
$$

where the $F_{i}$ are the faces of $A$ with Euclidean unit normals $f_{i}$ and the $X_{j}$ are the faces of $B^{0}$ with Euclidean unit normals $x_{j}$.

REMARK 2.15. These results also extend to more general surfaces $\Sigma$ in $\mathscr{X}$, where we have by a similar argument

$$
\mu_{B}(\Sigma)=\frac{1}{2 \varepsilon_{n-1}} \int_{\Sigma} \int_{\Sigma^{\prime}=\hat{o} B^{0}}|\widetilde{x}(\widetilde{g})| d \Sigma_{g}^{\prime} d \Sigma_{x} .
$$

There is another integral formula which may be derived from Lemma 2.9 .

Proposition 2.16. Let $f$ be a Euclidean unit vector in $\mathscr{P}^{*}$ and assume $B$ and $B^{0}$ are sufficiently smooth. Then, if $\Gamma$ is the $(n-2)$-dimensional set $\left\{g \in \partial B^{0} \mid f(\widetilde{g})=0\right\}$ parameterized by $\alpha_{1}, \alpha_{2}, \cdots$, $\alpha_{n-2}$,

$$
\begin{aligned}
\sigma\left(f^{\perp}\right)= & \pm \frac{1}{(n-1) \varepsilon_{n-1}} f \cdot \\
& \int_{I}\left[g, \frac{\partial g}{\partial \alpha_{1}}, \frac{\partial g}{\partial \alpha_{2}}, \cdots, \frac{\partial g}{\partial \alpha_{n-2}}\right] d \alpha_{1} d \alpha_{2}, \cdots, d \alpha_{n-2}
\end{aligned}
$$


where $[\cdots]$ represents the $(n-1)$-fold cross product.

Proof. Let $\Sigma$ be that part of $\partial B^{0}$ on which $f \cdot \widetilde{g}=f(\widetilde{g}) \geqq 0$. ( $\widetilde{g}$ is the Euclidean unit normal to $\Sigma$ at $g$.) Then $\Gamma=\partial \Sigma$. Considering the integral $\beta$ on the right of (2.17) we have

$$
\begin{aligned}
\beta & =f \cdot \int_{\Gamma}\left[g, \frac{\partial g}{\partial \alpha_{1}}, \cdots, \frac{\partial g}{\partial \alpha_{n-2}}\right] d \alpha_{1} \cdots d \alpha_{n-2} \\
& =\int_{\partial \Sigma}\left|f, g, \frac{\partial g}{\partial \alpha_{1}}, \cdots, \frac{\partial g}{\partial \alpha_{n-2}}\right| d \alpha_{1} \cdots d \alpha_{n-2}
\end{aligned}
$$

where $|\cdots|$ is the determinant. Expanding by cofactors of the first and second rows:

$$
\begin{aligned}
\beta= & \int_{\partial \Sigma} \sum_{i=1}^{n}(-1)^{i+1} \dot{\phi}_{i} \sum_{\substack{j=1 \\
j \neq i}}^{n}(-1)^{k_{i}, j} \psi_{j} \\
& \quad \times \frac{\partial\left(\psi_{1}, \psi_{2}, \cdots, \hat{\psi}_{i}, \cdots, \hat{\psi}_{j}, \cdots, \psi_{n}\right)}{\partial\left(\alpha_{1}, \alpha_{2}, \cdots, \alpha_{n-2}\right)} d \alpha_{1} \cdots d \alpha_{n-2} \\
= & \int_{\partial \Sigma} \sum_{i=1}^{n}(-1)^{i+1} \phi_{i} \sum_{\substack{j=1 \\
j \neq i}}^{n}(-1)^{k_{i}, j} \psi_{j} d \psi_{1} d \psi_{2} \cdots \widehat{d \psi_{i}} \cdots \widehat{d \psi_{j}} \cdots d \psi_{n}
\end{aligned}
$$

where $f=\left(\phi_{1}, \phi_{2}, \cdots, \phi_{n}\right), g=\left(\psi_{1}, \psi_{2}, \cdots, \psi_{n}\right)$ and $k_{i, j}=j+1$ if $j<i$ and $j$ if $j>i$. If we now apply Stokes' theorem ([11], p. 337; [17]) we get

$$
\begin{aligned}
\beta & = \pm \int_{\Sigma} d\left(\sum_{i=1}^{n}(-1)^{i+1} \phi_{i} \sum_{\substack{j=1 \\
j \neq i}}^{n}(-1)^{k_{i}, j} \psi_{j} d \psi_{1} \cdots \widehat{d \psi_{i}} \cdots \widehat{d \psi_{j}} \cdots d \psi_{n}\right) \\
& = \pm \int_{\Sigma} \sum_{i=1}^{n}(-1)^{i+1} \phi_{i} \sum_{\substack{j=1 \\
j \neq i}}^{n}(-1)^{k_{i}, j} d \psi_{j} d \psi_{1} \cdots \widehat{d \psi_{i}} \cdots \widehat{d \psi_{j}} \cdots d \psi_{n} \\
& = \pm \int_{\Sigma} \sum_{i=1}^{n}(-1)^{i+1} \phi_{i} \sum_{\substack{j=1 \\
j \neq i}}^{n} d \psi_{i} d \psi_{2} \cdots \widehat{d \psi_{i}} \cdots d \psi_{n} \\
& = \pm(n-1) \int_{\Sigma} \sum_{i=1}^{n} \phi_{i}(-1)^{i+1} d \psi_{1} d \psi_{2} \cdots \widehat{d \psi_{i}} \cdots d \psi_{n} \\
& = \pm(n-1) \int_{\Sigma} f \cdot \widetilde{g} d \Sigma= \pm(n-1) \int_{\Sigma}|f(\widetilde{g})| d \Sigma
\end{aligned}
$$

where the sign depends on the relative orientations of $\Sigma$ and $\Gamma$. By the symmetry of $B^{0}$, we have $\int_{\Sigma^{\prime}=\delta B^{0}}|f(\widetilde{g})| d \Sigma^{\prime}=2 \int_{\Sigma}|f(\widetilde{g})| d \Sigma$ and, with (2.13), $\sigma\left(f^{\perp}\right)=1 / \varepsilon_{n-1} \int|f(\widetilde{g})| d \Sigma= \pm\left(1 /(n-1) \varepsilon_{n-1}\right) \beta$ as required.

REMARK 2.17. In the case when $n=3$ the formula (2.17) reduces to 


$$
\sigma\left(f^{\perp}\right)=\frac{1}{2 \pi} f \cdot \int_{\Gamma} g \times d g
$$

when $\Gamma$ is oriented positively with respect to $f$.

We have shown that Definition 2.3 yields an area function $\gamma$ with the property that the surface area of a ball and that of the dual ball are equal. While we have not been able to show that $\gamma$ is uniquely determined by Axioms 2.2 and Equation 2.7, the following result is a step in that direction.

THEOREM 2.18. If $\gamma^{*}$ is an area function defined on all 2dimensional balls satisfying Axioms 2.2. and if $\mu_{A}^{*}(\partial A)=\mu_{A^{\circ}}^{*}\left(\partial A^{0}\right)$ for all 3-dimensional balls $A$, then $\gamma^{*}(B)=\gamma^{*}\left(B^{0}\right)$ for each unit ball $B$ in $\mathscr{R}^{2}$.

Proof. It is sufficient to consider the case when $B$ is a polygon. Let $A$ be a cylinder in $\mathscr{R}^{3}$ based on $B$. Then $A^{0}$ is a double cone based on $B^{\circ}$. We have

$$
\mu_{A^{0}}^{*}\left(\partial A^{0}\right)=\mu_{A}^{*}(\partial A)=\frac{1}{2} \mu_{B}^{*}(\partial B) \gamma^{*}(Q)+2 \gamma^{*}(B)
$$

where $Q$ is a parallelogram. Next, let $C$ be the ball obtained from $A$ by adding two conical caps each $1 / 2$ the height of $A$. Then $C^{0}$ is $A^{0}$ with the cones truncated at $1 / 2$ their height. (See Figure 2.1 for a simple case of $A, A^{0}, C$ and $C^{0}$.) Consider $\partial C^{0}$ which consists

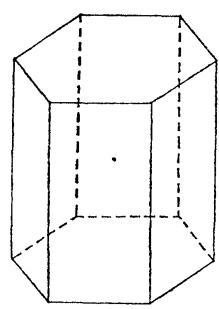

$A$

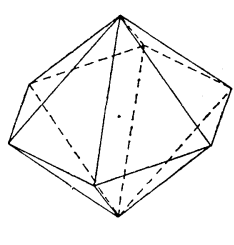

$A^{0}$

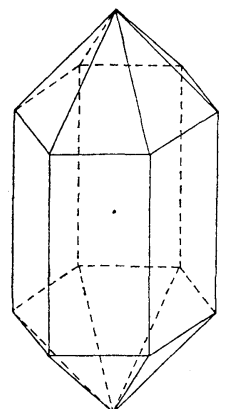

C

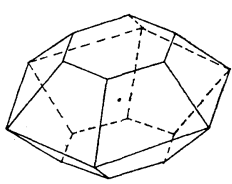

$C^{0}$

FIGURE 2.1

of two faces similar to $B^{0}$ and $1 / 2$ the size, and a number of trapezoids each obtained from a triangular face of $A^{0}$ by truncation at $1 / 2$ the height (and thus of area $3 / 4$ that of the triangle). Note that the cross-sections parallel to these faces in $C^{0}$ and $A^{0}$ are the same. Thus 


$$
\mu_{\sigma^{0}}^{*}\left(\partial C^{0}\right)=\frac{3}{4} \mu_{A^{0}}^{*}\left(\partial A^{0}\right)+\frac{1}{2} \gamma^{*}\left(B^{0}\right) .
$$

Now consider $\partial C$. The Minkowski area of each cap is still $\gamma^{*}(B)$ but now each rectangular face has a cross-section equivalent to a regular hexagon $H$ and of Euclidean area 3/2 times that of the corresponding rectangle in $A$. It is shown in $\S 6$ (Equation 6.2) that $\gamma^{*}(H)=(9 / 8) \gamma^{*}(Q)$ and hence

$$
\mu_{\sigma}^{*}(\partial C)=2 \gamma^{*}(B)+\frac{1}{3} \mu_{B}^{*}(\partial B) \gamma^{*}(H)=2 \gamma^{*}(B)+\frac{3}{8} \mu_{B}^{*}(\partial B) \gamma^{*}(Q) .
$$

Equating $\mu_{\sigma}^{*}(\partial C)$ and $\mu_{\sigma^{0}}^{*}\left(\partial C^{0}\right)$ and substituting $\mu_{A^{\circ}}^{*}\left(\partial A^{0}\right)$ from (2.19) we get

$$
\frac{3}{8} \mu_{B}^{*}(\partial B) \gamma^{*}(Q)+2 \gamma^{*}(B)=\frac{3}{8} \mu_{B}^{*}(\partial B) \gamma^{*}(Q)+\frac{3}{2} \gamma^{*}(B)+\frac{1}{2} \gamma^{*}\left(B^{0}\right)
$$

from which $\gamma^{*}(B)=\gamma^{*}\left(B^{0}\right)$ follows.

It does not seem easy to extend this result to higher dimensions, partly because the relationships between the analogues of the square $Q$ and the hexagon $H$ are not so easily established. We also do not know if Theorem 2.18 establishes uniqueness for the 2-dimensional function $\gamma$.

3. The function $\rho$. Let $\mathscr{P}$ be an $n$-dimensional Minkowski space with unit ball $B$ which generates, by Definition 2.3 and (2.1), a measure $\mu$ on $m$-dimensional subsets of $\mathscr{X}$.

DeFinition 3.1. For each measurable $m$-dimensional subset $\Omega$ of $\mathscr{X}$ let

$$
\rho(\Omega)=\mu(\Omega) \varepsilon_{m} m !
$$

where, as usual, $\varepsilon_{m}=\varepsilon\left(E_{m}\right)=\pi^{m / 2} / \Gamma(m+2 / 2)$.

In this section we show that $\rho$ has a number of remarkable properties with respect to Cartesian products.

If $\mathscr{X}$ and $\mathscr{Y}$ are $n$ and $m$-dimensional Minkowski spaces with unit balls $A$ and $B$ respectively, then $A \times B$ is a centrally symmetric convex body in $\mathscr{P} \times \mathscr{Y}$ which we may use as the unit ball. We also define a dual product (or "suspension") $A * B$ by taking $A * B$ to be the (closed) convex hull of the union of the natural embeddings of $A$ and $B$ into $\mathscr{X} \times \mathscr{Y}$. It is a straightforward computation to verify that $(A \times B)^{0}$ is equivalent to $A^{0} * B^{0}$ and thus the space $\mathscr{X}^{*} \times \mathscr{Y}^{*}$ with ball $A^{0} * B^{0}$ is dual to $\mathscr{X} \times \mathscr{Y}$ with ball $A \times B$. In 
the simple case $m=1, A \times B$ is the cylinder over $A$ and $A^{0} * B^{0}$ is the suspension of $A^{0}$. Choose a Euclidean metric in $\mathscr{X} \times \mathscr{Y}$ so that $\mathscr{X} \times\{0\}$ and $\{0\} \times \mathscr{Y}$ are orthogonal. It is well known that $\varepsilon(A \times B)=\varepsilon(A) \varepsilon(B)$. We need a similar result for $\varepsilon(A * B)$ which (as we could find no reference) we establish here.

Proposition 3.2. Let $A$ and $B$ be convex bodies about 0 in $\mathscr{E}^{n}$ and $\mathscr{E}^{m}$ respectively, then

$$
\varepsilon(A * B)=\varepsilon(A) \varepsilon(B) \frac{n ! m !}{(n+m) !} .
$$

Proof. By continuity, it is sufficient to consider the case when $A$ and $B$ are polytopes with extreme points $\left\{a_{1}, a_{2}, \cdots, a_{k_{1}}\right\}$ and $\left\{b_{1}\right.$, $\left.b_{2}, \cdots, b_{k_{2}}\right\}$ respectively. We will use $(x, 0)$ and $(0, y)$ to denote the points $\{x\} \times\{0\}$ and $\{0\} \times\{y\}$ in $\mathscr{E}^{n+m}$ when $x \in \mathscr{E}^{n}, y \in \mathscr{E}^{m}$. Similarly, if $U \subseteq \mathscr{E}^{n}, V \leqq \mathscr{E}^{m},(U, 0)$ and $(0, V)$ are the sets $U \times\{0\}$ and $\{0\} \times$ $V$.

By definition

$$
\begin{gathered}
A * B=\operatorname{co}\{(A, 0) \cup(0, B)\}=\operatorname{co}\left\{\left(a_{i}, 0\right),\left(0, b_{j}\right) \mid i=1,2, \cdots, k_{1},\right. \\
\left.j=1,2, \cdots, k_{2}\right\} .
\end{gathered}
$$

The polytope $A$ may be subdivided into simplices $S_{1}, S_{2}, \cdots, S_{l_{1}}$ which have a common vertex at 0 and which have only proper faces in common. Similarly, $B$ may be decomposed into simplices $T_{1}, T_{2}, \cdots, T_{l_{2}}$. Now $S_{i} * S_{j} \subseteq A * B$ and therefore $\bigcup_{i, j} S_{i} * S_{j} \subseteq A * B$. Conversely, let $x \in A * B$. Then $x=\sum_{i} \alpha_{i}\left(a_{i}, 0\right)+\sum_{j} \beta_{j}\left(0, b_{j}\right)$ where $\sum_{i} \alpha_{i}+\sum_{j} \beta_{j}=1, \alpha_{i}, \beta_{j} \geqq 0$. Let $\lambda=\sum_{i} \alpha_{i}$ so that $\sum_{j} \beta_{j}=1-\lambda$. Then, if $\lambda \neq 0,1$,

$$
x=\lambda\left(\sum_{i} \frac{\alpha_{i}}{\lambda}\left(a_{i}, 0\right)\right)+(1-\lambda)\left(\sum_{j} \frac{\beta_{j}}{1-\lambda}\left(0, b_{j}\right)\right) ;
$$

but $\sum_{i} \alpha_{i} / \lambda\left(a_{i}, 0\right) \in(A, 0)$ and hence is in some $\left(S_{i}, 0\right)$. Similarly $\sum_{j} \beta_{j} /(1-\lambda)\left(0, b_{j}\right) \in\left(0, T_{j}\right)$ for some $j$ and we have $x \in S_{i} * T_{j}$. If $\lambda=0$ then $x \in\left(0, T_{j}\right)$ for some $j$ and if $\lambda=1, x \in\left(S_{i}, 0\right)$, thus in all cases $x \in S_{i} \times T_{j}$. Therefore

$$
A * B=\bigcup_{i, \jmath} S_{i} * T_{j}
$$

Next note that the projections of $S_{i} * T_{j}$ onto $S_{i}$ and $T_{j}$ always carry interior points into interior points. Thus, if $S_{i} * T_{j}$ and $S_{i^{\prime}} * T_{j^{\prime}}$ have an interior point in common, we must have $i=i^{\prime}$ and $j=j^{\prime}$, and the decomposition (3.2) has no proper intersections. Therefore

$$
\varepsilon(A * B)=\varepsilon\left(\bigcup_{i, j} S_{i} * T_{j}\right)=\sum_{i, j} \varepsilon\left(S_{i} * T_{j}\right)
$$


and the proof of (3.1) is reduced to showing that

$$
\varepsilon(S * T)=\varepsilon(S) \varepsilon(T) \frac{n ! m !}{(n+m) !}
$$

for $n$ and $m$-dimensional simplices with a common vertex at 0 .

This formula is clearly true for $n=m=1$. We proceed by induction on $n$ for a fixed $m$ (this is sufficient by the symmetry of the expression in $n$ and $m$ ). Suppose $\left\{0, v_{1}, v_{2}, \cdots, v_{n}\right\}$ are the vertices of $S$ and $\left\{0, u_{1}, u_{2}, \cdots, u_{m}\right\}$ are the vertices of $T$. Then $S * T$ is the simplex in $\mathscr{E}^{n+m}$ with vertices $\left\{(0,0),\left(v_{k}, 0\right),\left(0, u_{l}\right)\right\} \mid k=$ $1, \cdots, n, l=1, \cdots, m\}$. If we let $S^{\prime}=\operatorname{co}\left\{0, v_{1}, \cdots, v_{n-1}\right\}$ then

$$
S * T=\operatorname{co}\left\{S^{\prime} * T \cup\left\{\left(v_{n}, 0\right)\right\}\right\} \text { and } S=\operatorname{co}\left\{S^{\prime} \cup\left\{V_{n}\right\}\right\} \text {. }
$$

Now, in Euclidean space, the $n$-dimensional content of an $n$ dimensional simplex is $n^{-1} \varepsilon(F) \varepsilon\left(v_{F}, F\right)$ where $\varepsilon(F)$ is the $(n-1)$ dimensional content of a face $F$ and $\varepsilon\left(v_{F}, F\right)$ is the perpendicular distance from $F$ to the opposite vertex $v_{F}$. Thus

$$
\varepsilon(S * T)=(n+m)^{-1} \varepsilon\left(S^{\prime} * T\right) \varepsilon\left(\left(v_{n}, 0\right), S^{\prime} * T\right)
$$

and $\varepsilon(S)=n^{-1} \varepsilon\left(S^{\prime}\right) \varepsilon\left(v_{n}, S^{\prime}\right)$. But since $\mathscr{E}^{n}$ and $\mathscr{E}^{m}$ are orthogonal in $\mathscr{E}^{n+m}, \varepsilon\left(\left(v_{n}, 0\right), S^{\prime} * T\right)=\left(v_{n}, S^{\prime}\right)$ and hence

$$
\varepsilon(S * T)=\frac{n}{n+m} \frac{\varepsilon\left(S^{\prime} * T\right) \varepsilon(S)}{\varepsilon\left(S^{\prime}\right)} .
$$

By the induction hypothesis $\varepsilon\left(S^{\prime} * T\right)=\varepsilon\left(S^{\prime}\right) \varepsilon(T)((n-1) ! m ! /(n-1+m) !)$ and we get $\varepsilon(S * T)=\varepsilon(S) \varepsilon(T)(n ! m ! /(n+m) !)$ as required.

Theorem 3.3. If $A$ and $B$ are any two Minkowski unit balls, then

(a) $\rho(A)=\rho\left(A^{0}\right), \rho(\partial A)=\rho\left(\partial A^{0}\right)$;

(b) $\rho(A \times B)=\rho(A * B)=\rho(A) \rho(B)$;

(c) $\rho(\partial(A \times B))=\rho(\partial(A * B))=\rho(\partial A) \rho(B)+\rho(A) \rho(\partial B)$.

Proof. Let $n$ and $m$ be the dimensions of $A$ and $B$.

(a) We have $\rho(A)=\mu_{A}(A) \varepsilon_{n} n !=\gamma(A) \varepsilon_{n} n !=\gamma\left(A^{0}\right) \varepsilon_{n} n !=\rho\left(A^{0}\right)$ and $\rho(\partial A)=\mu_{A}(\partial A) \varepsilon_{n-1}(n-1) !=\mu_{A}^{\circ}\left(\partial A^{0}\right) \varepsilon_{n-1}(n-1) !=\rho\left(\partial A^{0}\right)$, where the central equality follows from Corollary 2.9.

(b) First,

$$
\begin{aligned}
\rho(A \times B) & =\gamma(A \times B) \varepsilon_{n+m}(n+m) !=\frac{\varepsilon(A \times B) \varepsilon\left((A \times B)^{0}\right)}{\varepsilon_{n+m}} \varepsilon_{n+m}(n+m) ! \\
& =\varepsilon(A \times B) \varepsilon\left(A^{0} * B^{0}\right)(n+m) ! .
\end{aligned}
$$

Then by Proposition 3.2, 


$$
\begin{aligned}
\rho(A \times B) & =\varepsilon(A) \varepsilon(B) \varepsilon\left(A^{0}\right) \varepsilon\left(B^{0}\right) \frac{n ! m !}{(n+m) !}(n+m) ! \\
& =\frac{\varepsilon(A) \varepsilon\left(A^{0}\right)}{\varepsilon_{n}} \varepsilon_{n} n ! \frac{\varepsilon(B) \varepsilon\left(B^{0}\right)}{\varepsilon_{m}} \varepsilon_{m} m !=\rho(A) \rho(B) .
\end{aligned}
$$

Finally, by (a),

$$
\rho(A * B)=\rho\left(\left(A^{*} B\right)^{0}\right)=\rho\left(A^{0} \times B^{0}\right)=\rho\left(A^{0}\right) \rho\left(B^{0}\right)=\rho(A) \rho(B) .
$$

(c) Again we assume, without loss of generality, that $A$ and $B$ are polytopes. A face $F$ of $A \times B$ is either of the form $F_{A} \times B$ or of the form $A \times F_{B}$ (where $F_{A}$ is a face of $A$ and $F_{B}$ is a face of $B$ ). We consider the former case. Let $A_{F}$ be the cross-section of $A$ parallel to $F_{A}$ and $(A \times B)_{F}$ the cross-section of $A \times B$ parallel to $F$. Then $(A \times B)_{F}=A_{F} \times B$. Thus

$$
\begin{aligned}
\rho(F) & =\rho\left(F_{A} \times B\right)=(n+m-1) ! \varepsilon_{n+m-1} \mu\left(F_{A} \times B\right) \\
& =(n+m-1) ! \varepsilon_{n+m-1} \frac{\mu\left((A \times B)_{F}\right)}{\varepsilon\left((A \times B)_{F}\right)} \varepsilon\left(F_{A} \times B\right) \\
& =\rho\left(A_{F} \times B\right) \frac{\varepsilon\left(F_{A} \times B\right)}{\varepsilon\left(A_{F} \times B\right)} \\
& =\frac{\varepsilon\left(F_{A}\right) \varepsilon(B)}{\varepsilon\left(A_{F}\right) \varepsilon(B)} \rho\left(A_{F}\right) \rho(B)=\rho\left(F_{A}\right) \rho(B) .
\end{aligned}
$$

Similarly, if $F=A \times B_{F}, \rho(F)=\rho(A) \rho\left(F_{B}\right)$. Summing over all faces of $A \times B$ we get

$$
\rho(\partial(A \times B))=\rho(\partial A) \rho(B)+\rho(A) \rho(\partial B) .
$$

Then, using (a),

$$
\begin{aligned}
\rho(\partial(A * B)) & =\rho\left(\partial(A * B)^{0}\right)=\rho\left(\partial\left(A^{0} \times B^{0}\right)\right) \\
& =\rho\left(\partial A^{0}\right) \rho\left(B^{0}\right)+\rho\left(A^{0}\right) \rho\left(\partial B^{0}\right) \\
& =\rho(\partial A) \rho(B)+\rho(A) \rho(\partial B) .
\end{aligned}
$$

CoROLlaRy 3.4. If $A^{k}$ denotes either the k-fold cartesian product or the k-fold dual product in $\mathscr{X}^{k}$, then $\rho\left(A^{k}\right)=\rho(A)^{k}$ and $\rho\left(\partial A^{k}\right)=$ $k \rho(\partial A) \rho(A)^{k-1}$.

Proof. The first part is an immediate consequence of Theorem 3.3(b) and the second follows from parts (b) and (c) by induction.

Theorem 3.3 allows us to calculate $\gamma(A)$ and $\mu_{A}(\partial A)$ for balls which can be constructed from products or suspensions. For example, if $C_{n}$ denotes the $n$-dimensional cube and $O_{n}$ its dual - the $n$-dimensional "octahedron", we have 


$$
\gamma\left(C_{n}\right)=\frac{\rho\left(C_{n}\right)}{n ! \varepsilon_{n}}=\frac{\rho\left(C_{1}\right)^{n}}{n ! \varepsilon_{n}}=\frac{4^{n}}{n ! \varepsilon_{n}}
$$

and

$$
\begin{aligned}
\mu_{C_{n}}\left(\partial C_{n}\right) & =\frac{\rho\left(\partial C_{n}\right)}{(n-1) ! \varepsilon_{n-1}}=\frac{\rho\left(\partial C_{1}^{n}\right)}{(n-1) ! \varepsilon_{n-1}} \\
& =\frac{n \rho\left(\partial C_{1}\right) \rho\left(C_{1}\right)^{n-1}}{(n-1) ! \varepsilon_{n-1}}=\frac{2^{2 n-1} n}{(n-1) ! \varepsilon_{n-1}} .
\end{aligned}
$$

Another, related function, of some interest is

$$
\omega(B)=\frac{\rho(\partial B)}{\rho(B)}=\frac{\varepsilon_{n-1}}{n \varepsilon_{n}} \frac{\mu_{B}(\partial B)}{\mu_{B}(B)} .
$$

Theorem 3.3 implies that $\omega(B)=\omega\left(B^{0}\right)$ and $\omega(A \times B)=\omega(A * B)=$ $\omega(A)+\omega(B)$. The following establishes certain bounds on $\omega$.

Proposition 3.5. If $B$ is an n-dimensional Minkowski unit ball, then

$$
\frac{1}{2} \leqq \omega(B) \leqq \frac{n}{2}
$$

with equality on the right if $B$ is a cube or an "octahedron".

Proof. As usual we may assume $B$ is strictly convex and smooth. Now let $x$ be a point on $\partial B$ and consider $x^{0} \in \partial B^{0}$, and $\boldsymbol{P}_{x^{0}}\left(B^{0}\right)=\boldsymbol{P}_{\tilde{x}}\left(B^{0}\right)$ where the projection is taken onto the subspace orthogonal to $x^{0}$. Let $\Gamma=\left\{g \in \partial B^{0} \mid \boldsymbol{P}_{x^{0}}(g) \in \partial \boldsymbol{P}_{x^{0}}\left(B^{0}\right)\right\}$ and take $\boldsymbol{P}=$ $\operatorname{co}\left\{\Gamma \cup\left\{x^{0},-x^{0}\right\}\right\}$ and $P^{*}=\operatorname{co}\left\{\boldsymbol{P}_{x^{0}}\left(B^{0}\right) \cup\left\{x^{0},-x^{0}\right\}\right\}$. It is fairly easy to see that $\varepsilon(P)=\varepsilon\left(P^{*}\right)$. But $\varepsilon\left(P^{*}\right)=(2 / n) \varepsilon\left(\boldsymbol{P}_{x^{0}}\left(B^{0}\right)\right) \varepsilon\left(x^{0}\right)=2 \varepsilon\left(\boldsymbol{P}_{x^{0}}\left(B^{0}\right)\right) /$ $n \varepsilon(x)$ as $\varepsilon\left(x^{0}\right) \varepsilon(x)=1$. Thus, noting that $P \cong B^{0}$, we have

$$
\varepsilon\left(B^{0}\right) \geqq \varepsilon(P)=\varepsilon\left(P^{*}\right)=\frac{2 \varepsilon\left(\boldsymbol{P}_{x^{0}}\left(B^{0}\right)\right)}{n \varepsilon(x)} .
$$

Next, looking at the cylinder $C$ over $\boldsymbol{P}_{x^{0}}\left(B^{0}\right)$ with generator $2 x^{0}$, we see that $\varepsilon\left(B^{0}\right) \leqq \varepsilon(C) \leqq 2 \varepsilon\left(P_{x^{0}}\left(B^{0}\right)\right) \varepsilon\left(x^{0}\right)=2 \varepsilon\left(P_{x^{0}}\left(B^{0}\right)\right) / \varepsilon(x)$. This, with (3.4) yields

$$
\frac{1}{2} \varepsilon\left(B^{0}\right) \varepsilon(x) \leqq \varepsilon\left(\boldsymbol{P}_{x^{0}}\left(B^{0}\right)\right) \leqq \frac{n}{2} \varepsilon\left(B^{0}\right) \varepsilon(x) .
$$

Integrating (3.5) over $x \in \partial B$, we get

$$
\begin{array}{r}
\frac{1}{2} \varepsilon\left(B^{0}\right) \int_{\partial B} \varepsilon(x) d \Sigma_{x} \leqq \int_{\partial B} P_{x}\left(B^{0}\right) d \Sigma_{x} \\
=\varepsilon_{n-1} \mu_{B}(\partial B) \leqq \frac{n}{2} \varepsilon\left(B^{0}\right) \int_{\partial B} \varepsilon(x) d \Sigma_{x}
\end{array}
$$


But $\int_{\partial B} \varepsilon(x) d \Sigma_{x}=n \varepsilon(B)$, and thus, using (2.9),

$$
\frac{n \varepsilon_{n}}{2} \mu_{B}(B) \leqq \varepsilon_{n-1} \mu_{B}(\partial B) \leqq \frac{n^{2} \varepsilon_{n}}{2} \mu_{B}(B)
$$

which is equivalent to (3.3). Also, if $C_{n}$ is the $n$-dimensional cube,

$$
\omega\left(C_{n}\right)=\frac{\rho\left(\partial C_{n}\right)}{\rho\left(C_{n}\right)}=\frac{2^{2 n-1} n}{4^{n}}=\frac{n}{2} .
$$

4. The function $\sigma$ and the isoperimetric problem. The function $\sigma\left(f^{\perp}\right)$, defined by (2.2) as the ratio of Minkowski to Euclidean area in $f^{-1}(0)$, is important theoretically in connection with the solution to the isoperimetric problem. This seems to have first been recognized by $H$. Busemann [3], [4] who was able to give a complete solution to the isopermetric problem in a Minkowski space in terms of this function. For completeness, we outline his construction here.

The first step is to modify the definition of $\sigma$ to make it positive homogeneous rather than invariant under scalar multiples.

Let $\mathscr{X}$ be a Minkowski space with an "area" function defined on it, and let $\mathscr{X}$ and $\mathscr{X}^{*}$ be equipped with dual Euclidean norms. Define the function $\sigma^{\perp}$ on $\mathscr{Z}^{*}$ as follows:

$$
\sigma^{\perp}(f)=\left\{\begin{array}{cll}
0 & \text { if } & f=0 \\
\sigma\left(f^{\perp}\right)=\frac{\mu\left(B \cap f^{-1}(0)\right)}{\varepsilon\left(B \cap f^{-1}(0)\right)} & \text { if } & \varepsilon(f)=1 \\
\varepsilon(f) \sigma\left(\left(\frac{f}{\varepsilon(f)}\right)^{\perp}\right) & \text { if } & \varepsilon(f) \neq 0,1 .
\end{array}\right.
$$

Since $B$ is a convex body, $\sigma^{\perp}(f) \geqq 0$ with equality if and only if $f=0$. Clearly $\sigma^{\perp}(\alpha f)=|\alpha| \sigma^{\perp}(f)$, thus, if $\sigma^{\perp}$ is convex, it will be a norm on $\mathscr{X}^{*}$. Let $K=\left\{f \mid \sigma^{\perp}(f) \leqq 1\right\}$ and $I=K^{0} \cong \mathscr{X}$. The key step in Busemann's argument [3] is in showing that if $\sigma$ is convex and analytic and if $\Sigma$ is an analytic, closed, convex surface bounding a convex body $C$, then

$$
\mu(\Sigma)=\int_{\Sigma} \sigma^{\perp}(\widetilde{x}) d \Sigma_{x}=n \varepsilon_{\{1}(C, I)
$$

where $\varepsilon_{(1)}$ denotes the Euclidean mixed volume:

$$
n \varepsilon_{(1)}(C, I)=\lim _{\lambda \rightarrow 0} \lambda^{-1}(\varepsilon(C+\lambda I)-\varepsilon(C)) .
$$

Since arbitrary convex surfaces may be approximated by analytic ones, formula (4.2) holds for arbitrary compact, convex bodies $C$ 
with boundary $\Sigma$ (the integral being taken over that subset of $\Sigma$ where a unique tangent hyperplane exists), and for arbitrary convex $\sigma^{\perp}$.

THeOREM 4.1 (H. Busemann [3], [4]). If $\sigma^{\perp}$ is a convex function on $\mathscr{X}^{*}$ with unit ball $K$, then $I=K^{0}$ is the solution to the isoperimetric problem in $\mathscr{X}$ in the sense that surfaces $\Sigma$ which are homothetic translates of the boundary of $I$, and only these surfaces, minimize the surface area containing a given volume $\alpha$.

Proof. By (4.2) we have

$$
\int_{\Sigma} \sigma^{\perp}(\widetilde{x}) d \Sigma_{x}=n \varepsilon_{(1)}(C, I),
$$

also, we have Minkowski's inequality which states that

$$
\left(\varepsilon_{(1)}(C, I)\right)^{n} \geqq \varepsilon(C)^{n-1} \varepsilon(I)
$$

with equality if and only if $C$ is a homothetic translate of $I$. Hence, if $C$ has prescribed volume $\alpha$,

$$
\mu(\Sigma)=\int_{\Sigma} \sigma^{1}(\widetilde{x}) d \Sigma_{x} \geqq n \alpha^{n-1 / n} \varepsilon(I)^{1 / n}
$$

with equality only for homothetic translates of $I$.

DeFINITION 4.2. The isoperimetrix $\hat{I}$ in a Minkowski space $(\mathscr{X}, \mu)$ is that homothetic translate of $I$ centered at the origin and satisfying

$$
\mu(\partial \hat{I})=n \mu(\hat{I})
$$

(where $n$ is the dimension of $\mathscr{C}$ ).

REMARKS (a) In Theorem 4.1 it was assumed that $\alpha$ represents Euclidean volume. In fact, since any two measures of volume are related by a scalar multiple, the result is independent of the particular measure used. The surface bounding the greatest Euclidean volume also bounds the greatest Minkowski volume.

(b) On the other hand, the body $I$, and thus $\hat{I}$, depend heavily on the measure of surface area which is determined by the function $\sigma^{\perp}$ and ultimately by the choice of the function $\gamma$.

(c) Equation (4.2) is important, not only because it yields Theorem 4.1, but because it generalizes Minkowski's method of calculating surface area (see Proposition 4.4 below).

(d) Busemann showed that his definition of $\gamma$ (2.5) leads to a function $\sigma^{\perp}$ which is convex (although he also generalized Theorem 
4.1 to the nonconvex case). He, however, gave no specific example, and even when the unit ball is a simple 3-dimensional object such as a cube or octahedron, the body $I$ which is generated is not simple to describe. If $\gamma$ is taken to be the function defined by (2.6), sample calculations show that $\sigma^{\perp}$ is, in general, not convex. In contrast with these rather negative results, the use of $\gamma$ as defined by (2.8) or (2.9) yields some rather pleasing results.

For the remainder of this section, we will assume that $\gamma$ is given by (2.8) and thus $\sigma$ is given by (2.12).

LEMMA 4.3. If $\hat{I}$ is the isoperimetrix in the n-dimensional Minkowski space $(\mathscr{X}, \mu)$ with unit ball $B$, then, for a Euclidean norm on $\mathscr{X}, \mathscr{X}^{*}$ we can construct $I$ as above and

$$
\hat{I}=\frac{\varepsilon_{n}}{\varepsilon\left(B^{0}\right)} I
$$

Proof. Let $\hat{I}=\lambda I$, then $\varepsilon(\hat{I})=\lambda^{n} \varepsilon(I)$ and hence

$$
\mu(\hat{I})=\varepsilon(\hat{I}) \frac{\mu(B)}{\varepsilon(B)}=\lambda^{n} \varepsilon_{n}^{-1} \varepsilon(I) \varepsilon\left(B^{0}\right) .
$$

We also have $\mu(\partial \hat{I})=\lambda^{n-1} \mu(\partial I)$, using (4.2) and (4.4) we get

$$
\mu(\hat{I})=n^{-1} \mu(\partial \hat{I})=n^{-1} \lambda^{n-1} \mu(\partial I)=\lambda^{n-1} \varepsilon_{(1)}(I, I)=\lambda^{n-1} \varepsilon(I) .
$$

Equating this with (4.5) yields $\lambda=\varepsilon_{n} / \varepsilon\left(B^{0}\right)$.

REMARK. From the definition of $\gamma$, we see that $\lambda=\varepsilon_{n} / \varepsilon\left(B^{0}\right)=$ $\varepsilon(B) / \mu(B)$.

This last expression for $\lambda$ is contained in the work of $H$. Busemann [3].

Proposition 4.4 (Busemann [3]). If $C$ is a convex body in a Minkowski space then

$$
\mu(\partial C)=\lim _{\alpha \rightarrow 0} \frac{\mu(C+\alpha \hat{I})-\mu(C)}{\alpha}=n \mu_{(1)}(C, \hat{I}) .
$$

Proof. Rewriting (4.2) and (4.3) we have

$$
\mu(\partial C)=\lim _{\alpha \rightarrow 0} \frac{\varepsilon(C+\alpha I)-\varepsilon(C)}{\alpha}=\lim _{\alpha \rightarrow 0} \frac{\varepsilon\left(C+\alpha \lambda^{-1} \widehat{I}\right)-\varepsilon(C)}{\alpha}
$$

where, by Lemma $4.3 \lambda^{-1}=\mu(B) / \varepsilon(B)$. Thus 


$$
\begin{aligned}
\mu(\partial C) & =\lim _{\alpha \rightarrow 0} \frac{\varepsilon(C+\alpha \hat{I})-\varepsilon(C)}{\alpha \lambda} \\
& =\lim _{\alpha \rightarrow 0} \frac{\mu(C+\alpha \hat{I})-\mu(C)}{\alpha} .
\end{aligned}
$$

We now come to the main result of this section.

THEOREM 4.5. If the unit ball $B$ in the Minkowski space ( $\mathscr{X}$, $\mu)$ is a convex polytope, then the isopermetrix $\hat{I}$ is also a convex polytope.

CoROLlary 4.6. The function $\sigma^{\perp}$ as defined in (4.1) is convex in any Minkowski space.

Before proceeding with the proof of Theorem 4.5 we first need

LEMMA 4.7. Let $\mathscr{X}$ be a hyperplane in $\mathscr{E}^{n}$ and let $h$ denote the pole of $\mathscr{H}$ with respect to the unit ball. If $\mathscr{Y}$ is an $(n-1)$ dimensional subspace, then the pole of $\mathscr{C} \cap \mathscr{Y}$ in $\mathscr{Y}$ is $\boldsymbol{P}(h)$ where $\boldsymbol{P}$ is the orthogonal projection of $\mathscr{E}^{n}$ onto $\mathscr{Y}$.

Proof. Let $u_{1}, u_{2}, \cdots, u_{n-1}$ be an orthonormal basis for $\mathscr{Y}$ and extend this, by the addition of $u_{n}$, to an orthonormal basis for $\mathscr{E}^{n}$. Let $\mathscr{H}$ be determined by the equation

$$
\left(\alpha_{1}, \alpha_{2}, \cdots, \alpha_{n}\right) \cdot x=1
$$

in this coordinate system. Then $h=\left(\alpha_{1}, \alpha_{2}, \cdots, \alpha_{n}\right)$. $\mathscr{C} \cap \mathscr{Y}$ has the equation $\left(\alpha_{1}, \alpha_{2}, \cdots, \alpha_{n-1}\right) \cdot x=1$ in the space $\mathscr{Y}$. Thus the pole of this hyperplane in $\mathscr{Y}$ is $\left(\alpha_{1}, \alpha_{2}, \cdots, \alpha_{n-1}\right)$ which is clearly $\boldsymbol{P}(h)$.

Proof of Theorem 4.5. For the proof we rely on the work of H. Busemann and E.G. Straus [5], and, in particular, $\S 3$ of that paper.

Let $\mathscr{X}, \mathscr{X}^{*}$ be equipped with dual Euclidean norms, and we will identify $\mathscr{X}$ and $\mathscr{E}^{n}$. We will assume that each vertex of $B$ is the intersection of precisely $n$ linearly independent faces. (It is always possible to approximate $B$ arbitrarily closely by such a polytope with a fixed number of faces.)

Let $\mathscr{Y}$ be a subspace of dimension $n-2$ which does not contain a vertex of $B$ and extend a basis $u_{3}, u_{4}, \cdots, u_{n}$ of $\mathscr{Y}$ to a basis $u_{1}, u_{2}, \cdots, u_{n}$ of $\mathscr{X}$. Let $\left(\beta_{1}, \beta_{2}, \cdots, \beta_{n}\right)$ be coordinates with respect to this basis and let $\mathscr{C}_{\theta}$ be the ("rotating") hyperplane determined by 


$$
\cos \theta \beta_{1}+\sin \theta \beta_{2}=0
$$

where $\theta$ ranges from 0 to $2 \pi$.

Consider $\mathscr{H}_{\theta}$ in a position which does not intersect a vertex of $B$. We wish to calculate the Euclidean area of the polar reciprocal of the intersection of $\mathscr{H}_{\theta}$ and $B$. Let $\alpha_{i 1} \beta_{1}+\alpha_{i 2} \beta_{2}+\cdots \alpha_{i n} \beta_{n}=1$ be the equation of a typical face of $B$ which intersects $\mathscr{H}_{\theta}$. Then the polar of this face is $a_{i}=\left(\alpha_{i 1}, \alpha_{i 2}, \cdots, \alpha_{i n}\right)$. Thus, by Lemma 4.6, the pole (in $\mathscr{H}_{\theta}$ ) of the intersection of this face with $\mathscr{H}_{\theta}$ is given by

$$
\boldsymbol{P}_{\theta}\left(a_{i}\right)=\left(\alpha_{i 1}-\lambda_{i} \cos \theta, \alpha_{i 2}-\lambda_{i} \sin \theta, \alpha_{i 3}, \cdots, \alpha_{i n}\right)
$$

where $\boldsymbol{P}_{\theta}$ is the orthogonal projection on $\mathscr{K}_{\theta}$ and $\lambda_{i}$ is such that $\boldsymbol{P}_{\theta}\left(a_{i}\right) \in \mathscr{K}_{\theta}$.

For $i=1,2, \cdots, n-1$ let the faces of $B$ be chosen so that their poles in $\mathscr{H}_{\theta}$ form the vertices of a face of $\left(B \cap \mathscr{H}_{\theta}\right)^{0}$. Then the $(n-1)$-dimensional Euclidean content of the simplex subtended at the origin by this face is

$$
\kappa_{n} \varepsilon\left(\left[\boldsymbol{P}_{\theta}\left(a_{1}\right), \boldsymbol{P}_{\theta}\left(a_{2}\right), \cdots, \boldsymbol{P}_{\theta}\left(a_{n-1}\right)\right]\right)
$$

where $\kappa_{n}$ is a constant depending only on $n$. However, each $\boldsymbol{P}_{\theta}\left(a_{i}\right)$ lies in $\mathscr{C}_{\theta}$ and thus $\left[\boldsymbol{P}_{\theta}\left(a_{1}\right), \cdots, \boldsymbol{P}_{\theta}\left(a_{n-1}\right)\right]$ is a multiple of the unit normal $x_{\theta}=(\cos \theta, \sin \theta, 0, \cdots, 0)$ to $\mathscr{H}_{\theta}$. Thus

$$
\begin{aligned}
& \varepsilon\left(\left[\boldsymbol{P}_{\theta}\left(a_{1}\right), \cdots, \boldsymbol{P}_{\theta}\left(a_{n-1}\right)\right]\right)= \pm x_{\theta} \cdot\left[\boldsymbol{P}_{\theta}\left(a_{1}\right), \cdots, \boldsymbol{P}_{\theta}\left(a_{n-1}\right)\right] \\
& = \pm\left|\begin{array}{llllc}
\cos \theta & \sin \theta & 0 & 0 & \cdots 0 \\
\alpha_{11}-\lambda_{1} \cos \theta & \alpha_{12}-\lambda_{1} \sin \theta & \alpha_{13} & \alpha_{14} & \alpha_{1 n} \\
\alpha_{21}-\lambda_{2} \cos \theta & \alpha_{22}-\lambda_{2} \sin \theta & \alpha_{23} & \alpha_{24} & \alpha_{2 n} \\
\vdots & & & & \\
\vdots & & & & \\
\alpha_{n-1,1}-\lambda_{n-1} \cos \theta & \alpha_{n-1,2}-\lambda_{n-1} \sin \theta & \alpha_{n-1,3} & \alpha_{n-1,4} & \cdots \alpha_{n-1, n}
\end{array}\right| \\
& = \pm\left|\begin{array}{cccccc}
\cos \theta & \sin \theta & 0 & 0 & \cdots & 0 \\
\alpha_{11} & \alpha_{12} & \alpha_{13} & \alpha_{14} & \cdots & \alpha_{1 n} \\
\alpha_{21} & \alpha_{22} & \alpha_{23} & \alpha_{24} & \cdots & \alpha_{2 n} \\
\vdots & & & & & \vdots \\
\alpha_{n-1,1} & \alpha_{n-1,2} & \alpha_{n-1,3} & \alpha_{n-1,4} & \cdots & \alpha_{n-1, n}
\end{array}\right| \\
& = \pm x_{\theta} \cdot\left[a_{1}, a_{2}, \cdots, a_{n-1}\right] \text {. }
\end{aligned}
$$

If we assume that the faces of $B$ are numbered in such a way as to make the determinants positive, then we get 


$$
\begin{aligned}
\varepsilon\left(\left(B \cap \mathscr{C}_{\theta}\right)^{0}\right) & =\kappa_{n} \sum_{j} x_{\theta} \cdot\left[a_{1}^{j}, a_{2}^{j}, \cdots, a_{n-1}^{j}\right] \\
& =\kappa_{n} x_{\theta} \cdot \sum_{j}\left[a_{1}^{j}, a_{2}^{j}, \cdots, a_{n-1}^{j}\right] \\
& =\kappa_{n} x_{\theta} \cdot\left(\sigma_{1}, \sigma_{2}, \cdots, \sigma_{n}\right) \\
& =\kappa_{n}\left(\cos \theta \sigma_{1}+\sin \theta \sigma_{2}\right)=\sigma\left(x_{\theta}\right)
\end{aligned}
$$

where

$$
\left(\sigma_{1}, \sigma_{2}, \cdots, \sigma_{n}\right)=\sum_{j}\left[a_{1}^{j}, a_{2}^{j}, \cdots, a_{n-1}^{j}\right]
$$

and the summation is extended over all 1-dimensional edges of $B$ which intersect $\mathscr{K}_{\theta}$.

We now follow the construction described by Busemann and Straus in [5]. Let $\mathscr{P}$ be the 2-dimensional plane spanned by the first two coordinates $u_{1}, u_{2}$. The subspace $\beta_{1}=\beta_{2}=0$ is a hyperplane in $\mathscr{H}_{\theta}$ and, on one side of this hyperplane construct the point $y$ in $\mathscr{P} \cap \mathscr{C}$ such that $\varepsilon(y)=1 / \sigma\left(x_{\theta}\right)=1 /\left(\cos \theta \sigma_{1}+\sin \theta \sigma_{2}\right)$. Then $y=$ $\left(\cos \theta \sigma_{1}+\sin \theta \sigma_{2}\right)^{-1}(\sin \theta,-\cos \theta, 0 \cdots 0)=\left(\gamma_{1}, \gamma_{2}, 0, \cdots, 0\right)$ where $\gamma_{1}=$ $\sin \theta / \cos \theta \sigma_{1}+\sin \theta \sigma_{2}$ and $\gamma_{2}=-\cos \theta / \cos \theta \sigma_{1}+\sin \theta \sigma_{2}$. Thus $\gamma_{1}, \gamma_{2}$ satisfy the equation $\sigma_{2} \gamma_{1}-\sigma_{1} \gamma_{2}=1$. As $\sigma_{1}$ and $\sigma_{2}$ depend only on the 1-dimensional edges of $B$ which intersect $\mathscr{K}_{\theta}$, they are constant provided $\mathscr{K}_{\theta}$ does not intersect a vertex of $B$. Hence the curve traced out in $\mathscr{P}$ by $y$ consists of a finite number of straight line segments. This implies, see [5], that the solution to the isoperimetric problem is a polytope which will be convex if and only if the polygon traced out by $y$ in $\mathscr{P}$ is convex. To show this we need to investigate what happens when $\mathscr{K}_{0}$ intersects a vertex of $B$.

Let $v$ be the intersection of $n$ faces with normals $\left(\alpha_{i 1}, \alpha_{i 2}, \cdots\right.$, $\left.\alpha_{i n}\right) i=1,2, \cdots, n$. A vector along any of the $n 1$-dimensional edges of $B$ which meet at $v$ is given by the cross-product of $(n-1)$ of these normals. As we saw above, these same cross-products enter into the calculation of $\left(\sigma_{1}, \sigma_{2}, \cdots, \sigma_{n}\right)$ and, there, were ordered so that their inner product with $x_{\theta}$ was positive. Before $\mathscr{K}_{\theta}$ crosses $v$ it cuts some $k$ of these $n$ edges and after it has crossed $v$ it cuts the remaining $n-k$ edges. We may suppose that we have chosen $x_{\theta}$ (or equivalently, a side of $\beta_{1}=\beta_{2}=0$ in $\mathscr{C}_{\theta}$ ) in such a way that before $\mathscr{H}_{\theta}$ crosses $v$ the cross-products in $\left(\sigma_{1}, \cdots, \sigma_{n}\right)$ point away from $v$ which means that after $\mathscr{H}_{\theta}$ crosses $v$ the vectors along the $n-k$ edges point toward $v$. Therefore, to calculate the change in the vector $\left(\sigma_{1}, \cdots, \sigma_{n}\right)$ due to $\mathscr{H}_{\theta}$ crossing $v$ we need to subtract the cross-products which determine vectors along the $k$ edges away from $v$ and add the cross-products which determine vectors along the $n-k$ edges toward $v$. We wish to determine what this sum is. 
Let $a_{1}, a_{2}, \cdots, a_{n}$ be an ordering of the normals so that $D=$ $\left|a_{1}, a_{2}, \cdots, a_{n}\right|>0$. Then, to say that $\left[a_{1}^{\prime}, a_{2}^{\prime}, \cdots, a_{i-1}^{\prime}, a_{i+1}^{\prime}, \cdots, a_{n}^{\prime}\right]$ where $\left\{a_{1}^{\prime}, \cdots, a_{i-1}^{\prime}, a_{i+1}^{\prime}, \cdots, a_{n}^{\prime}\right\}$ is some ordering of $\left\{a_{1}, \cdots, a_{i-1}\right.$, $\left.a_{i+1}, \cdots, a_{n}\right\}$ is directed towards $v$ is to say that $a_{i} \cdot\left[a_{1}^{\prime}, \cdots, a_{i-1}^{\prime}\right.$, $\left.a_{i+1}^{\prime}, \cdots, a_{n}^{\prime}\right]>0$. That is, using a common notation standard in 3space, we require

$$
\left[a_{1}^{\prime}, \cdots, a_{i-1}^{\prime}, a_{i+1}^{\prime}, \cdots, a_{n}^{\prime}\right]=\left|a_{1}, a_{i}, \cdots, a_{i-1}, u, a_{i+1}, \cdots, a_{n}\right|
$$

where $u$ is the "vector" $\left(u_{1}, u_{2}, \cdots, u_{n}\right)$. But now, if we solve the $n$ linear equations

$$
a_{i} \cdot z=1 \quad(i=1,2, \cdots, n)
$$

to obtain the coefficients of the vector $v$, it is a matter of elementary linear algebra, using Cramer's rule, to see that

$$
\sum_{i=1}^{n}\left[a_{1}^{\prime}, a_{2}^{\prime}, \cdots, a_{i-1}^{\prime}, a_{i+1}^{\prime}, \cdots, a_{n}^{\prime}\right]=D v .
$$

Thus the change in $\left(\sigma_{1}, \sigma_{2}, \cdots, \sigma_{n}\right)$ due to crossing the vertex $v$ in this direction is to add a positive multiple of $v$. $\mathscr{H}_{0}$ of course also crosses the vertex $-v$, but in the opposite direction, thus the addition for $-v$ is also $D v$.

We are now in a position to say exactly what happens in the plane $\mathscr{P}$ when $\mathscr{H}_{0}$ crosses one or more vertices and their negatives.

First, as $\sigma_{1}$ and $\sigma_{2}$ can only change when $\mathscr{C}_{\theta}$ crosses a vertex, the vertices of the polygon in $\mathscr{P}$ are positive multiples of the projections in $\mathscr{P}$ of the vertices of $B$. Second, as $\mathscr{C}_{\theta}$ passes one or more vertices the new values $\sigma_{1}^{\prime}, \sigma_{2}^{\prime}$ of $\sigma_{1}, \sigma_{2}$ are given by $\sigma_{1}^{\prime}=$ $\sigma_{1}+\delta_{1}, \sigma_{2}^{\prime}=\sigma_{2}+\delta_{2}$ where $\left(\delta_{1}, \delta_{2}, 0, \cdots, 0\right)$ is the appropriate multiple of the corresponding vertex of the polygon. The normals (in $\mathscr{P}$ ) to the edges on either side of this vertex are $\left(\sigma_{2},-\sigma_{1}\right)$ and $\left(\sigma_{2}^{\prime}-\sigma_{1}^{\prime}\right)$. The polygon is convex at this vertex if and only if the angle between these normals lies between 0 and $\pi$, i.e., if and only if the sine of this angle is positive, or $-\sigma_{2} \sigma_{1}^{\prime}+\sigma_{1} \sigma_{2}^{\prime}>0$. But $-\sigma_{2} \sigma_{1}^{\prime}+$ $\sigma_{1} \sigma_{2}^{\prime}=-\sigma_{2}\left(\sigma_{1}+\delta_{1}\right)+\sigma_{1}\left(\sigma_{2}+\delta_{2}\right)=\sigma_{1} \delta_{2}-\sigma_{2} \delta_{1}$. This last expression, however, is a positive multiple of the sine of the angle between $\left(\sigma_{1}, \sigma_{2}\right)$, which is directed along the side of the polygon away from the vertex, and the vertex. As this is positive the proof is complete.

REMARKS (a). The proof of Theorem 4.5 actually gives us a method for constructing the isoperimetrix when the unit ball is a polytope. It is an easy matter to see that the points $\left(\sigma_{1}, \cdots, \sigma_{n}\right)$ constructed in the proof are the vertices of a convex polytope which must be a multiple of the isoperimetrix, the correct multiple 
being determined by (4.4). (See examples in $\S 6$.)

(b) It also follows that the edges of the isoperimetrix are parallel to the vertices of the unit ball.

When $B$ is strictly convex, we can use Proposition 2.16 to derive an integral representation ((4.9), Theorem 4.8) for $I$ which is similar to the formula (4.7) in the polytope case. To this end, let $f \in \mathscr{X}^{0}$ and define $\Sigma^{f}=\left\{g \in \partial B^{0} \mid f(\widetilde{g}) \geqq 0\right\}$ and $\Gamma_{f}=\left\{g \in \partial B^{0} \mid f(\widetilde{g})=0\right\}$. is smooth and thus the Euclidean unit normal $\widetilde{g}$ at $g$ is well defined and continuous.) If we now define

$$
z(f)=\frac{1}{\varepsilon_{n-1}} \int_{\Sigma f} \widetilde{g} d \Sigma_{g}^{f}
$$

we have, as in the proof of Proposition 2.16,

$$
z(f)=\frac{1}{(n-1) \varepsilon_{n-1}} \int_{\Gamma}\left[g, \frac{\partial g}{\partial \alpha_{1}}, \cdots, \frac{\partial g}{\partial \alpha_{n-2}}\right] d \alpha_{1} \cdots d \alpha_{n-2}
$$

where $\Gamma$ is a suitably oriented $(n-2)$ manifold lying in $\Gamma_{f}$.

THEOREM 4.8. If $B$ is strictly convex and $z(f)$ is defined as above, then $\partial I=\left\{z(f) \mid f \in \mathscr{X}^{*}\right\}=\{z(f) \mid f \in \partial K\}$. Moreover, $f^{-1}(1)$ is a hyperplane supporting $I$ at $z(f)$ when $f \in \partial K$.

Proof. It is clear from (4.8) and the definition of $\Sigma^{f}$ that

$$
\begin{aligned}
f(z(f))=f \cdot z(f) & =\frac{1}{\varepsilon_{n-1}} \int_{\Sigma f} f(\widetilde{g}) d \Sigma_{g}^{f} \geqq \frac{1}{\varepsilon_{n-1}} \int_{\Sigma} f(\widetilde{g}) d \Sigma_{g} \\
& =f \cdot\left(\frac{1}{\varepsilon_{n-1}} \int_{\Sigma} \widetilde{g} d \Sigma_{g}\right)
\end{aligned}
$$

where $\Sigma$ in any other subset of $\partial B^{0}$ for which the integral exists, and that equality obtains only if $\Sigma$ and $\Sigma^{f}$ differ by a subset of $\Gamma_{f}$. In particular $f \cdot z(f) \geqq f \cdot z(g)$. Now, from (4.1), we see that

$$
\begin{aligned}
& \sigma^{\perp}(f)=\varepsilon(f) \sigma\left(\left(\frac{f}{\varepsilon(f)}\right)^{\perp}\right)=\varepsilon(f)\left(\frac{f}{\varepsilon(f)}\right) \\
& \cdot \frac{1}{(n-1) \varepsilon_{n-1}} \int_{\Gamma}\left[g, \frac{\partial g}{\partial \alpha_{1}}, \cdots, \frac{\partial g}{\partial \alpha_{n-2}}\right] d \alpha_{1}, \cdots, d \alpha_{n-2}=f \cdot z(f) .
\end{aligned}
$$

Thus $K=\{f \mid f \cdot z(f) \leqq 1\}$ and $\partial K=\{f \mid f \cdot z(f)=1\}$. For any $f \in K$, and any $g, f \cdot z(g) \leqq f \cdot z(f) \leqq 1$ which implies that $z(g) \in K^{0}=I$ for all $g \in \mathscr{Q}^{*}$. But if $f \in \partial K, 1=f \cdot z(f) \geqq f \cdot x$ for any $x \in I$. Thus $f$ supports $I$ at $z(f)$ and $z(f) \in \partial I$ (and $z(f)$ supports $K$ at $f$ ). Now $B^{\circ}$ is smooth and thus $z(f)$ is continuous as a function of $f$ implying that $K$ is also smooth. Hence $I$ is strictly convex. Let $x \in \partial I$. Then there is an $f \in \partial K$ such that $f^{-1}(1)$ supports $I$ at $x$. Clearly 
$f^{-1}(1) \cap I=\{x\}$. But $f^{-1}(1)$ supports $I$ at $z(f)$, thus $x=z(f)$ and $\partial I=\{z(f) \mid f \in \partial K\}$ as required.

Corollary 4.9. If $B$ is strictly convex then $I$ (and $\hat{I}$ ) is also strictly convex.

CoRollary 4.10. If $B$ is strictly convex and smooth then $I(\hat{I})$ is also.

Proof. Suppose $I$ is not smooth. Then there is a point $x=$ $z(f)=z(g)$ on the boundary of $I$ with two distinct supporting functionals $f \neq g, f, g \in \partial K$. As $f$ and $g$ cannot be multiples of each other, there is an open (relative to $\partial B^{0}$ ) set $\Lambda \cong \Sigma^{g} \sim \Sigma^{f} \subseteq \Gamma_{f}$ with Euclidean normals perpendicular to $f$ which is impossible as $B^{0}$ must be strictly convex.

REMARK. If $B$ is not strictly convex the integral representation may still be used to advantage if we evaluate (4.9) where it is defined and add in the cross-products corresponding to (4.7) at the isolated singular points. With this modification, $z(f)$ is defined for almost all $f$ and we have $I=\overline{\mathrm{co}}\{z(f) \mid f \in \partial K\}$ as every exposed point of $I$ is a $z(f)$ for some $f$.

If the space $\mathscr{X}$ is 2 -dimensional, then $\sigma^{\perp}$ and consequently $I$ do not depend on the definition of "area" used. It can be shown that, if $\mathscr{X}$ and $\mathscr{X}^{*}$ are identified by identifying a pair of dual Euclidean bases, $I=U B^{0}$ where $U$ is a rotation by $\pi / 2$ (in either direction). This leads to two special properties of the isoperimetrix $\hat{I}$ in this case.

THEOREM 4.11. If $\mathscr{X}$ is 2-dimensional, then $\mu(\hat{I})=\pi$.

Proof. From Lemma 4.3, $\hat{I}=\left(\pi / \varepsilon\left(B^{0}\right)\right) I$, and thus

$$
\mu(\hat{I})=\frac{\varepsilon(\hat{I}) \varepsilon\left(B^{0}\right)}{\pi}=\frac{\pi^{2} \varepsilon(I) \varepsilon\left(B^{0}\right)}{\varepsilon\left(B^{0}\right)^{2} \pi}=\frac{\varepsilon(I)}{\varepsilon\left(B^{0}\right)} \pi=\pi .
$$

CoRollary 4.12. If $A$ and $B$ are two unit balls $i \pi \mathscr{R}^{2}$ and if $\beta$ is any positive real number, then $\varepsilon\left(A+\beta \widehat{I}_{B}\right) \varepsilon\left(B^{0}\right)=\varepsilon\left(B^{0}+\beta \widehat{I}_{A^{0}}\right) \varepsilon(A)$ and hence $\mu_{B}\left(A+\beta \hat{I}_{B}\right)=\mu_{A^{0}}\left(B^{0}+\beta \hat{I}_{A^{0}}\right)$.

Proof. It follows from (4.2) and Theorem 2.11 that, for any two balls $A$ and $B, \varepsilon_{(1)}\left(A, \hat{I}_{B}\right) \varepsilon\left(B^{0}\right)=\varepsilon_{(1)}\left(B^{0}, \hat{I}_{A^{0}}\right) \varepsilon(A)$. In 2-dimensions, we also have $\varepsilon\left(\hat{I}_{B}\right) \varepsilon\left(B^{0}\right)=\pi \mu_{B}\left(\hat{I}_{B}\right)=\pi^{2}=\varepsilon\left(\hat{I}_{A^{0}}\right) \varepsilon(A)$. Thus if we expand 
$\varepsilon\left(A+\beta \widehat{I}_{B}\right) \varepsilon\left(B^{0}\right)=\varepsilon(A) \varepsilon\left(B^{0}\right)+\beta \varepsilon_{(1)}\left(A, \hat{I}_{B}\right) \varepsilon\left(B^{0}\right)+\beta^{2} \varepsilon\left(\hat{I}_{B}\right) \varepsilon\left(B_{0}\right)$ and $\varepsilon\left(B^{0}+\right.$ $\left.\beta \hat{I}_{A^{0}}\right) \varepsilon(A)=\varepsilon\left(B^{0}\right) \varepsilon(A)+\beta \varepsilon_{(1)}\left(B^{0}, \hat{I}_{A^{0}}\right) \varepsilon(A)+\beta^{2} \varepsilon\left(\hat{I}_{A^{0}}\right) \varepsilon(A)$ and compare coefficients, the result follows.

5. Normality of points and functions. In this section we consider some results related to the ideas of normality and transversality as defined by Busemann in [4] (see also [5]). Let $\mathscr{X}$ be a Minkowski space with unit ball $B$ and isoperimetrix $\hat{I}$. Let $\hat{\delta}$ be the norm on $\mathscr{X}$ associated with unit ball $\hat{I}$ and $\hat{K}, \hat{\sigma}$ be the dual ball and norm in $\mathscr{X}^{*}$. For an $x \in \mathscr{X}$ and an $f \in \mathscr{X}^{*}$ we define the Minkowski sine function $s m$ by:

$$
\operatorname{sm}(x, f)=\frac{f(x)}{\hat{\sigma}(f) \mu(x)} .
$$

It is not hard to show that, if $\mathscr{L}$ is the line $\{\alpha x \mid \alpha \in R\}$, $|s m(x, f)|=\overline{s m}\left(\mathscr{L}, f^{-1}[1]\right)$ where $\overline{s m}$ is the sine function defined in [4].

Following [4], we set

$$
\begin{aligned}
& \alpha(f)=\sup _{x \in \mathscr{x}} s m(x, f)=\frac{\mu(f)}{\hat{\sigma}(f)} \\
& \alpha(x)=\sup _{f \in \mathscr{C}^{*}} \sin (x, f)=\frac{\hat{\delta}(x)}{\mu(x)}
\end{aligned}
$$

and define:

(a) $x$ is normal to $f$ and $f$ is transversal to $x$ if $\operatorname{sm}(x, f)=\alpha(f)$.

(b) $x$ is transversal to $f$ and $f$ is normal to $x$ if $\operatorname{sm}(x, f)=$ $\alpha(x)$.

From (5.1) and (5.2) it is clear that $x$ is normal to $f$ if and only if $f / \mu(f)$ supports $B$ at $x / \mu(x)$. Similarly, $x$ is transversal to $f$ if and only if $f / \hat{\sigma}(f)$ supports $\hat{I}$ at $x / \hat{\delta}(x)$. From these remarks and corollaries to Theorems 4.6 and 4.8 we have:

THEOREM 5.1.

(1) Given $x$, there is an $f$ transversal to $x$. $f$ is unique up to a multiple if $B$ is smooth.

(2) Given $x$, there is an $f$ normal to $x$. $f$ is unique up to a multiple if $B$ is smooth and strictly convex.

(3) Given $f$, there is an $x$ transversal to $f$. $x$ is unique up to a multiple if $B$ is strictly convex.

(4) Given $f$, there is an $x$ normal to $f$. $x$ is unique up to a multiple if $B$ is strictly convex.

CoROllaRY 5.2. If $B$ is strictly convex and $f \in \mathscr{Z}^{*}$, then $z(f)$ 
(as defined in (4.8)) is the unique (up to a multiple) transversal to $f$.

As a consequence of Theorem 5.1 (3) and 4.2 of [5], we have:

CoRollary 5.3. Hyperplanes minimize "area" in $\mathscr{X}$.

6. Examples. In this section we give a number of examples. They are of two types. First we have calculations in $\mathscr{R}^{3}$ that were originally made to see whether it was possible to satisfy the condition

$$
\mu_{A}(\partial A)=\mu_{A^{0}}\left(\partial A^{0}\right) .
$$

They are presented here, partly because of their intrinsic interest, but mainly as evidence that the function $\gamma$ as defined in (2.8) is the only area function which satisfies (6.1) as well as Axioms 2.1. The second set of examples are of specific isoperimetrices. As far as we know, no such examples have been given, in dimensions larger than 2 , for other area functions.

A. Examples in $R^{3}$. Through this part $\mathscr{X}$ will be $\mathscr{R}^{3}$ upon which we shall impose a variety of unit balls $B$.

EXAMPLE 6.1. Let $B$ be a cube, so that $B^{0}$ is a regular octahedron. The unit sphere $\partial B$ consists of 6 squares, each of which is parallel to a subspace whose unit ball (in the relative norm) is a square $Q$ of the same size and similarly oriented. Thus we have $\mu(\partial B)=6 \gamma(Q)$. On the other hand, $\partial B^{0}$ consists of 8 triangles each of which is parallel to a subspace whose unit ball is a regular hexagon $H$ of side $1 / 2$ that of the triangle. The area of each face is $2 / 3$ that of the hexagon, hence $\mu\left(\partial B^{0}\right)=(16 / 3) \gamma(H)$. Condition (6.1) then yields the relation

$$
\frac{\gamma(Q)}{8}=\frac{\gamma(H)}{9}
$$

which was used in the proof of Theorem 2.18.

EXAMPLe 6.2. Consider next, the ball $B$ (see Figure 6.1) which is a cube of side $\sqrt{2}$ with two pyramidal caps, the apex of each being $\sqrt{2} / 1+\alpha(0<\alpha<1)$ from the origin. The dual $B^{0}$ is thus a truncated octahedron. The cross-sections of $B$ parallel to the square faces are the hexagons $H_{\alpha}$ illustrated in Figure 6.2, while each triangular face is $1 / 4$ of the rectangular cross-section. 


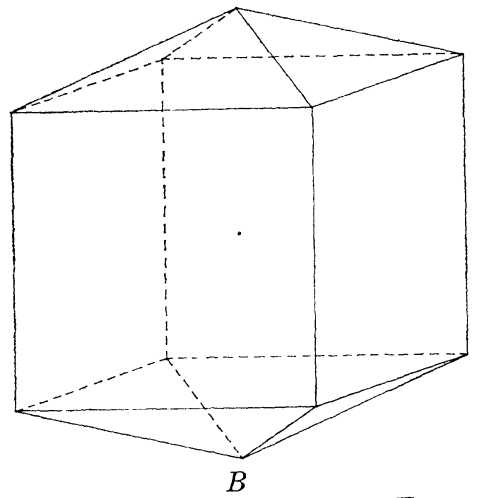

FIgURE 6.1

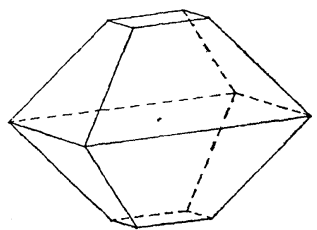

$B^{0}$

The cross-sections of $B^{0}$ parallel to the trapezoidal faces are regular hexagons $H$. Routine calculations then show that

$$
\mu(\partial B)=8 \frac{(1+\alpha)}{(3+\alpha)} \gamma\left(H_{\alpha}\right)+2 \gamma(Q)
$$

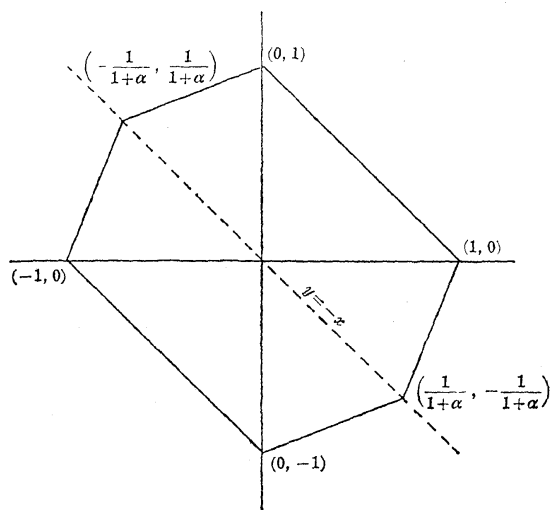

$H_{r}$

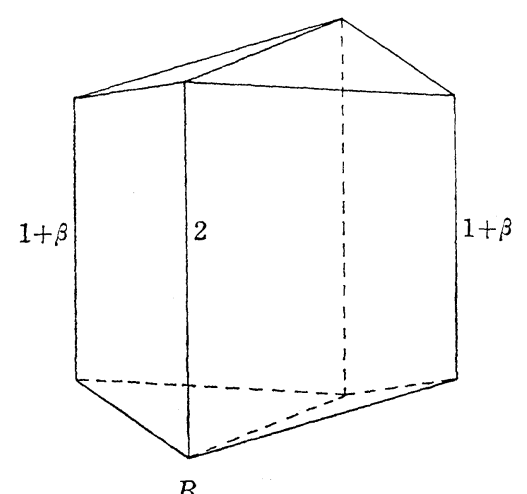

B

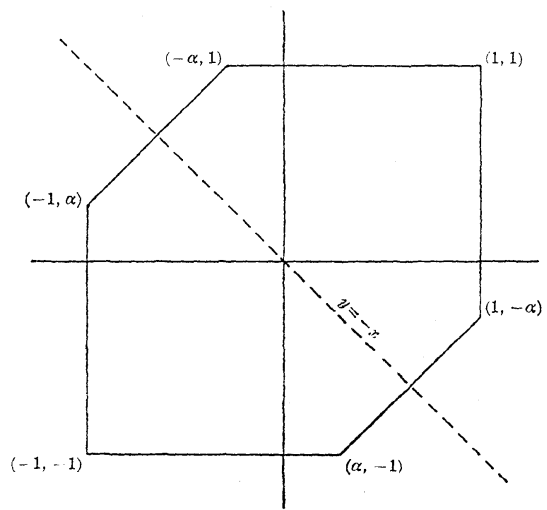

$H_{\alpha}^{0}$

FIGURE 6.2

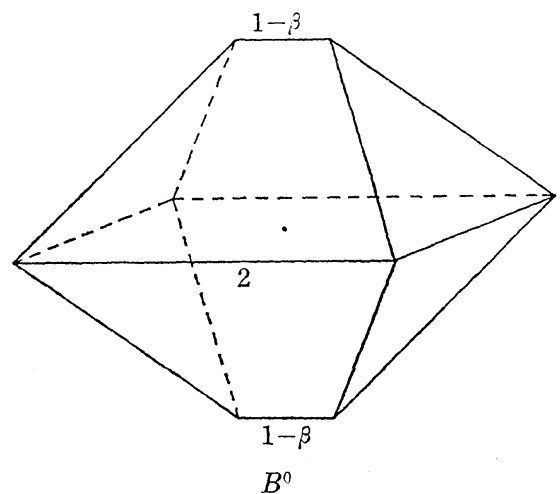

FIGURE 6.3 
and

$$
\mu\left(\partial B^{0}\right)=\frac{(1-\alpha)^{2}}{2} \gamma(Q)+\frac{4(3-\alpha)(1+\alpha)}{3} \gamma(H) .
$$

Equating, and using (6.2) we get

$$
\gamma\left(H_{\alpha}\right)=\frac{\left(9-\alpha^{2}\right)}{8} \gamma(Q)
$$

and applying Theorem 2.18;

$$
\gamma\left(H_{\alpha}^{0}\right)=\gamma\left(H_{-\alpha}\right)=\frac{9-\alpha^{2}}{8} \gamma(Q) .
$$

( $H_{\alpha}^{\prime \prime}$ is shown in Figure 6.2.)

EXAMPLE 6.3. Equation (6.4) may also be derived by considering the ball depicted in Figure 6.3. When $\beta=0$, this ball is especially interesting as it is an example of a self dual centrally symmetric polyhedron.

EXAMPLE 6.4. Let $D_{n}$ denote a regular $2^{n}$-gon and take $B$ to be a cylinder of height 2 on a copy of $D_{n-1}$ inscribed in the unit circle. Then $\mu(\partial B)=2 \gamma\left(D_{n-1}\right)+2^{n-1} \tan \left(\pi / 2^{n-1}\right) \gamma(Q)$. The dual ball $B^{0}$ is a double pyramid of height 2 on a copy of $D_{n-1}$ circumscribed about the unit circle. Each of the $2^{n}$ faces of $\partial B^{0}$ is a triangle of Euclidean area $\sqrt{2} \tan \left(\pi / 2^{n-1}\right)$ parallel to a certain cross-section $P_{n-1}$. (For large $n P_{n-1}$ is an approximation to two parabolic arcs.) If the Euclidean area of $P_{n-1}$ is $\varepsilon\left(P_{n-1}\right)$ then $\mu\left(\partial B^{0}\right)=2^{n} \sqrt{2} \tan \left(\pi / 2^{n-1}\right) \gamma\left(P_{n-1}\right) /$ $\varepsilon\left(P_{n-1}\right)$ and, using (6.1),

$$
\begin{gathered}
2^{n} \sqrt{2} \sin \left(\frac{\pi}{2^{n-1}}\right) \frac{\gamma\left(P_{n-1}\right)}{\varepsilon\left(P_{n-1}\right)}=2 \cos \left(\frac{\pi}{2^{n-1}}\right) \gamma\left(D_{n-1}\right) \\
+2^{n-1} \sin \left(\frac{\pi}{2^{n-1}}\right) \gamma(Q) .
\end{gathered}
$$

EXAMPLE 6.5. A further relationship between these same quantities is obtained by considering a somewhat more complicated situation. Consider $D_{n}$ with a copy of $D_{n-1}$ inscribed in it so that its vertices are the midpoints of each second edge of $D_{n}$. Then move copies of this $D_{n-1}$ both above and below $D_{n}$ and take $B$ to be the convex hull of the 3 polygones. See Figure 6.4 for the case $n=3$. The point of this construction is that the relevant cross-sections of $B$ are all rectangles, hexagons of the type $H_{\alpha}$ for $\alpha=2 \cos \left(\pi / 2^{n-1}\right)-1$, and $D_{n}$, while the cross-sections of $B^{0}$ parallel 


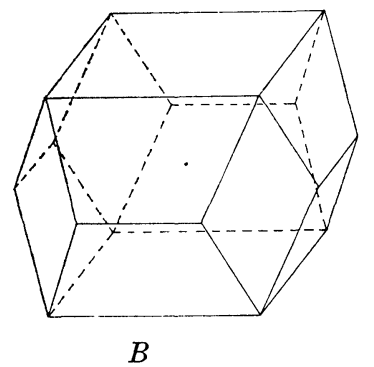

Figure 6.4

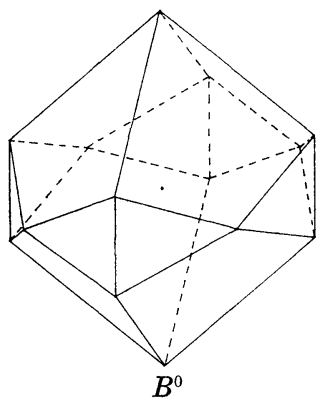

to faces are of the form $H_{\alpha}^{0}$ where $\alpha=\cos \left(\pi / 2^{n-1}\right)$ and the $P_{n-1}$ of the previous example.

Trigonometric calculations of the same type, but more complicated than before, yield, with (6.3) and (6.4), the equations:

$$
\begin{aligned}
\mu(\partial B)= & 2 \cos \left(\frac{\pi}{2^{n-1}}\right) \cos ^{2}\left(\frac{\pi}{2^{n}}\right) \gamma\left(D_{n}\right) \\
& +2^{n-2} \tan \left(\frac{\pi}{2^{n}}\right)\left[5-\cos ^{2}\left(\frac{\pi}{2^{n-1}}\right)\right] \gamma(Q) \\
\mu\left(\partial B^{0}\right)= & 2^{n \cdot \sqrt{2}} \sin \left(\frac{\pi}{2^{n-1}}\right) \frac{\gamma\left(P_{n-1}\right)}{\varepsilon\left(P_{n-1}\right)} \\
& +2^{n-2} \tan \left(\frac{\pi}{2^{n}}\right)\left[1-\cos \left(\frac{\pi}{2^{n-1}}\right)\right]\left[3+\cos \left(\frac{\pi}{2^{n-1}}\right)\right] \gamma(Q) .
\end{aligned}
$$

Using equations (6.1) and (6.5) we obtain, after considerable simplification,

$$
2 \cos \left(\frac{\pi}{2^{n-1}}\right)\left[\cos ^{2}\left(\frac{\pi}{2^{n}}\right) \gamma\left(D_{n}\right)-\gamma\left(D_{n-1}\right)\right]=0 .
$$

A straightforward inductive argument (noting that $D_{2}=Q$ ) shows that:

$$
\gamma\left(D_{n}\right)=2^{2 n-3} \sin ^{2}\left(\frac{\pi}{2^{n}}\right) \gamma(Q) .
$$

Letting $n \rightarrow \infty$ and using Axioms 2.1 (a) and (c) (so that $\lim _{n \rightarrow \infty} \gamma\left(D_{n}\right)=$ $\pi)$ we get

$$
\gamma(Q)=\frac{8}{\pi}
$$

This may now be substituted in (6.2), (6.3), (6.4) and (6.6) to give

$$
\gamma(H)=\frac{9}{\pi}
$$




$$
\begin{aligned}
\gamma\left(H_{\alpha}\right) & =\frac{9-\alpha^{2}}{\pi} \\
\gamma\left(D_{n}\right) & =\frac{2^{2 n} \sin ^{2}\left(\frac{\pi}{2^{n}}\right)}{\pi} .
\end{aligned}
$$

B. Examples of Isoperimetrices. In this part we compute, as indicated in Theorem 4.8 and the remarks following Theorems 4.5 and 4.8, the isoperimetrix for a number of three dimensional balls. As the calculations are similar in each case, they are given only for Example 6.6. The space $\mathscr{X}$ in each case is three dimensional and has been given a Euclidean norm.

EXAMPLE 6.6. Let $B$ be a regular octahedron with vertices at $( \pm 1,0,0),(0, \pm 1,0)$ and $(0,0, \pm 1) . \quad(\gamma(B)=8 / \pi)$. Let $f^{-1}(0)$ be a

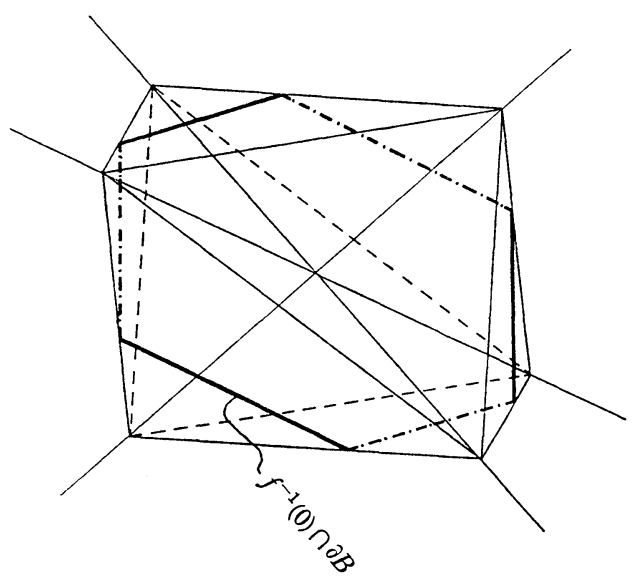

FIGURE 6.5

subspace where $f$ is in the first octant. Then this subspace intersects $\partial B$ in the faces with polars $(-1,1,1),(-1,1,-1),(1,1$, $-1),(1,-1,-1),(1,-1,1)$ and $(-1,-1,1)$ in order (see Figure 6.5). Thus the corresponding vertex of $I$ is given by the sum of the cross products of consecutive pairs of these polars. A simple computation yields $(-8,-8,-8)$. Similarly, taking $f$ in each octant, we see that the vertices of $I$ are $( \pm 8, \pm 8, \pm 8)$ and the isopermetrix is a cube. Setting $\mu(\partial(\lambda I))=3 \mu(\lambda I)$ we get $\lambda=1 / 12$ and thus $\hat{I}$ is the cube with vertices $( \pm(2 / 3), \pm(2 / 3), \pm 2 / 3)$.

EXAMPLE 6.7 . Let $B$ be the cube with vertices at $( \pm 1, \pm 1, \pm 1)$. $(\gamma(B)=8 / \pi)$. Then $\hat{I}$ is a rhombic-dodecahedron with vertices $( \pm 1$, $\pm 1, \pm 1),( \pm 2,0,0),(0, \pm 2,0)$ and $(0,0, \pm 2)$. 
EXAMPLE 6.8. Let $B$ be the double cone of height 2 over the unit circle. $(\gamma(B)=\pi)$. Then $\hat{I}$ is a cylinder of radius $8 / 3 \pi$ and height $4 / 3$.

EXAMPLE 6.9. Let $B$ be the cylinder of height 2 over the unit circle. $(\gamma(B)=\pi)$. Then $\hat{I}$ is the body obtained by rotating the area under the curve $x=4 / \pi \cos ((\pi / 4) z)$ about the $z$ axis.

7. Conjectures and problems. In this section we collect together a number of questions which, as far as we know, remain open.

Problem 7.1. For the given function $\gamma$, do affine sets minimize area?

This may be reformulated as a polyhedral inequality and, as such, was posed by Busemann and Shephard [6] (See also [5]). In this form, it may be stated as follows. In $\mathscr{R}^{n}$, let $P$ be a closed $m$-polytope with faces $F_{1}, F_{2}, \cdots, F_{k}$. Let $B$ be a (centrally symmetric) convex body in $\mathscr{R}^{n}$. Furthermore let $\boldsymbol{P}_{i}(i=1,2, \cdots, k)$ be the orthogonal projection onto the $m$-dimensional subspace parallel to the face $F_{i}$. Then Problem 7.1 is equivalent to showing that

$$
\varepsilon\left(\boldsymbol{P}_{1}(B)\right) \varepsilon\left(F_{1}\right) \leqq \sum_{i=2}^{k} \varepsilon\left(\boldsymbol{P}_{i}(B)\right) \varepsilon\left(F_{i}\right)
$$

Problem 7.2. Do Axioms 2.1 and the requirement that $\mu_{A}(\partial A)=$ $\mu_{A^{0}}\left(\partial A^{0}\right)$ uniquely determine $\gamma$ ?

We conjecture that the answer is yes. The examples in $\S 6 \mathrm{~A}$ show that $\gamma$ is uniquely determined on certain polygons. The question may be modified in many ways, for example, adding the requirement that $\gamma(B)=\gamma\left(B^{0}\right)$. This leads to

Problem 7.3. If $\mu_{A}(\partial A)=\mu_{A^{0}}\left(\partial A^{0}\right)$ for all $n$-dimensional balls, is it true that we must have $\gamma(B)=\gamma\left(B^{0}\right)$ for $(n-1)$-dimensional balls?

This is the $n$-dimensional generalization of Theorem 2.18.

If we define $I_{n}$ to be the map carrying the set of centrally symmetric closed convex $n$-dimensional bodies into itself which takes a unit ball $B$ onto its isoperimetrix $\hat{I}$, then there are a number of questions regarding $\boldsymbol{I}_{n}$.

Problem 7.4. Is $\boldsymbol{I}_{n}$ an injection? 
Problem 7.5. Is the Euclidean ball $E_{n}$ the only fixed point of $I_{n}$ for $n>2$ ?

Problem 7.6. If $n>2$, do the successive iterates $\left(\boldsymbol{I}_{n}\right)^{k}(B)$ converge (in some sense) to $E_{n}$ ?

This conjecture is supported by numerous calculations which seem to indicate that $I_{n}(B)$ is more "sphere like" than $B$.

Problem 7.7. Does $I_{n}$ map smooth balls to smooth sets?

We know from $\S 4$ that $I_{n}$ maps polytopes to polytopes, strictly convex balls to strictly convex sets, and smooth and strictly convex balls to similar sets.

The remaining problems deal with maximums and minimums of the various numerical functions involved.

Problem 7.8. What are the lower bounds for $\gamma(B)$ in $\mathscr{R}^{3}\left(\mathscr{R}^{n}\right)$ ?

As was stated in $\S 2$, it is known [1], [12] that in $\mathscr{R}^{2}, 8 / \pi \leqq$ $\gamma(B) \leqq \pi$ with equaiity on the left only for parallelograms and on the right only for ellipses.

Problem 7.9. What are the bounds for $\mu_{B}(\partial B)$ in $\mathscr{R}^{3}\left(\mathscr{R}^{n}\right)$ ?

It is known [13] that in $\mathscr{R}^{2}, 6 \leqq \mu_{A}(\partial A) \leqq 8$, with equality on the left only for regular hexagons and on the right only for parallelograms. In $\mathscr{R}^{3}$, if $A$ is the cubo-octahedron, (and thus $A^{0}$ is the rhombicdodecahedron) we know that $\mu_{A}(\partial A)=\mu_{A^{0}}\left(\partial A^{0}\right)=36 / \pi<$ $\mu_{E_{3}}\left(\partial E_{3}\right)=4 \pi$ and this is the smallest value we have found. (A truncated cone over a regular hexagon also gives the value $36 / \pi$.) If $C_{3}$ is the cube in $\mathscr{R}^{3}$ then $\mu_{C_{3}}\left(\partial C_{3}\right)=48 / \pi$. Is this maximal? Note, that in $\mathscr{R}^{n}$ the cube is not, in general, maximal as $\lim _{n \rightarrow \infty} \mu C_{n}\left(\partial C_{n}\right) /$ $\mu_{E_{n}}\left(\partial E_{n}\right)=0$.

Problem 7.10. What is the lower bound for $\omega(B)$ in $\mathscr{R}^{n}$ ?

\section{REFERENCES}

1. R. P. Bambah, Polar reciprocal convex bodies, Proc. Cambridge Phil. Soc., 51 (1955), 377-378.

2. R. V. Benson, Euclidean geometry and convexity, McGraw-Hill, New York, 1966, especially Chapter 6.

3. H. Busemann, The isoperimetric problem for Minkowski area, Amer. J. Math., 71 (1949), 743-762. 
4. H. Busemann, The foundations of Minkowski geometry, Comm. Mathematici Helvetici, 24 (1950), 156-187.

5. H. Busemann and E. G. Straus, Area and normality, Pacific J. Math., 10 (1960), $35-72$

6. H. Busemann and G. C. Shephard, Convexity on non-convex sets, Proc. Colloq. on Convexity, Copenhagen, 1965 (Copenhagen, 1967), 20-23 and p. 309.

7. J. W. S. Cassels, An Introduction to the Geometry of Numbers, Springer Verlag, Berlin, 1959.

8. H. G. Eggleston, Convexity (Cambridge Tracts in Mathematics and Mathematical Physics, No. 47), Cambrige, 1958.

9. K. Mahler, Ein Übertragungsprinzip für konvexe korper, Časopis Pest. Mat. Fyz., 68 (1939), 93-102.

10. P. McMullen and G. C. Shephard, Convex polytopes and the upper bound conjecture, London Math. Soc. Lecture Notes Series 3, Cambridge, 1971.

11. M. E. Munroe, Modern Multidimensional Calculus, Addison-Wesley, Reading, 1963.

12. L. A. Santaló, An affine invariant for convex bodies in n-dimensional space, (in Spanish), Portugaliae Math., 8 (1949), 155-161.

13. J. J. Schäffer, Inner diameter, perimeter and girths of spheres, Math. Ann., 173 (1967), 59-82.

14. — The self-circumference of polar convex discs, Arch. der Math., 24 (1973), 87-90.

15. J. J. Schäffer and K. Sundaresan, Reflexivity and the girth of the spheres, Math. Ann., 184 (1970), 163-168.

16. A. C. Thompson, An equiperimetric property of Minkowski circles, Bull. London Math. Soc., 7 (1975), 271-272.

17. H. Whitney, Geometry and Integration, Princeton, N. J., 1957.

Received January 13, 1977 and in revised form November 1, 1977. This work was supported in part by National Research Council of Canada Grant Number A-4066.

DALHOUSIE UNIVERSITY

Halifax, N. S., Canada 


\section{PACIFIC JOURNAL OF MATHEMATICS}

\section{EDITORS}

DONALD BABBITT (Managing Editor)

University of California

Los Angeles, California 90024

HUgo RossI

University of Utah

Salt Lake City, UT 84112

C. C. MOORE and ANDREW OGG

University of California

Berkeley, CA 94720

\section{J. DUGUNDJI}

Department of Mathematics University of Southern California Los Angeles, California 90007

R. Finn and J. Milgram Stanford University Stanford, California 94305

\section{ASSOCIATE EDITORS}

E. F. BECKENBACH

B. H. NEUMANN

F. WOLF

K. YoshidA

\section{SUPPORTING INSTITUTIONS}

UNIVERSITY OF BRITISH COLUMBIA CALIFORNIA INSTITUTE OF TECHNOLOGY UNIVERSITY OF CALIFORNIA MONTANA STATE UNIVERSITY UNIVERSITY OF NEVADA, RENO NEW MEXICO STATE UNIVERSITY OREGON STATE UNIVERSITY UNIVERSITY OF OREGON
UNIVERSITY OF SOUTHERN CALIFORNIA STANFORD UNIVERSITY UNIVERSITY OF HAWAII UNIVERSITY OF TOKYO UNIVERSITY OF UTAH WASHINGTON STATE UNIVERSITY UNIVERSITY OF WASHINGTON 


\section{Pacific Journal of Mathematics}

Vol. 85, No. $1 \quad$ September, 1979

Ralph Alexander, Metric averaging in Euclidean and Hilbert spaces...... 1

B. Aupetit, Une généralisation du théorème de Gleason-Kahane-Żelazko

pour les algèbres de Banach..............................

Lung O. Chung, Jiang Luh and Anthony N. Richoux, Derivations and

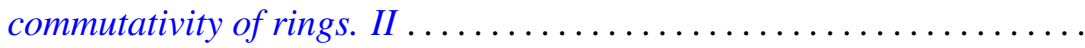

Lynn Harry Erbe, Integral comparison theorems for third order linear

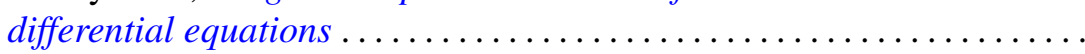

Robert William Gilmer, Jr. and Raymond Heitmann, The group of units of a

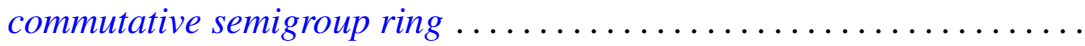

George Grätzer, Craig Robert Platt and George William Sands, Embedding lattices into lattices of ideals ...........................

Raymond D. Holmes and Anthony Charles Thompson, $n$-dimensional area and content in Minkowski spaces ....................... 77

Harvey Bayard Keynes and M. Sears, Modelling expansion in real flows....

Taw Pin Lim, Some classes of rings with involution satisfying the standard

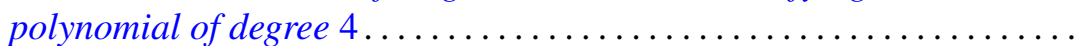

Garr S. Lystad and Albert Robert Stralka, Semilattices having bialgebraic congruence lattices ................................... 131

Theodore Mitchell, Invariant means and analytic actions . . . . . . . . . 145

Daniel M. Oberlin, Translation-invariant operators of weak type ........ 155

Raymond Moos Redheffer and Wolfgang V. Walter, Inequalities involving

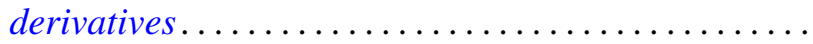

Eric Schechter, Stability conditions for nonlinear products and semigroups ................................

Jan Søreng, Symmetric shift registers ........................ 201

Toshiji Terada, On spaces whose Stone-Čech compactification is $\mathrm{Oz} \ldots \ldots .231$

Richard Vrem, Harmonic analysis on compact hypergroups ... 